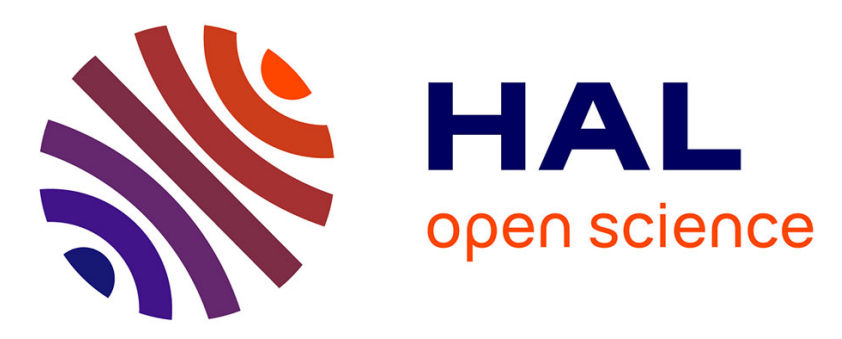

\title{
Colloidal molecules and patchy particles: complementary concepts, synthesis and self-assembly
}

Weiya Li, Hervé Palis, Rémi Merindol, Jérôme Majimel, Serge Ravaine, Etienne Duguet

\section{- To cite this version:}

Weiya Li, Hervé Palis, Rémi Merindol, Jérôme Majimel, Serge Ravaine, et al.. Colloidal molecules and patchy particles: complementary concepts, synthesis and self-assembly. Chemical Society Reviews, 2020, 49 (6), pp.1955-1976. 10.1039/C9CS00804G . hal-02558746

\author{
HAL Id: hal-02558746 \\ https://hal.science/hal-02558746
}

Submitted on 11 May 2020

HAL is a multi-disciplinary open access archive for the deposit and dissemination of scientific research documents, whether they are published or not. The documents may come from teaching and research institutions in France or abroad, or from public or private research centers.
L'archive ouverte pluridisciplinaire HAL, est destinée au dépôt et à la diffusion de documents scientifiques de niveau recherche, publiés ou non, émanant des établissements d'enseignement et de recherche français ou étrangers, des laboratoires publics ou privés. 


\title{
Colloidal molecules and patchy particles: complementary concepts, synthesis and self-assembly
}

Received 00th January 20xx, Accepted 00th January 20xx

DOI: $10.1039 / \times 0 \times x 00000 x$

\begin{abstract}
Weiya Li, ${ }^{a, b}$ Hervé Palis, ${ }^{a, b}$ Rémi Mérindol, ${ }^{b}$ Jérôme Majimel, ${ }^{a}$ Serge Ravaine ${ }^{b}$ and Etienne Duguet*a
This review describes the latest advances in the synthesis and assembly of specific colloids such as the colloidal molecules as defined by van Blaaderen in 2003 and the patchy particles imagined a few years later. The two concepts are closely related because some may serve as precursors of others and vice versa. To best mimic the molecular structures, it is necessary to introduce the notions of directed binding and valence which result in the concept of patches arranged on the particle surface according to the conventional repulsion figures. The assembly of patchy particles has made it possible to reconstitute molecules and macromolecules of simple geometry. But the existence of extended assemblies of larger dimensions has been demonstrated mostly by simulation and it struggles experimentally with the purity of the batches of building blocks.
\end{abstract}

\section{Introduction}

Colloidal self-assembly is a relevant alternative to chemical synthesis route and top-down fabrication techniques for building increasingly complex structures and materials. The first building units were isotropic spherical particles from sizemonodisperse batches. These particles, often called colloidal atoms (CAs), helped to clarify crystallization and phase transition mechanisms, because, thanks to their mesoscopic size, their dynamics are slowed down sharply in comparison with atomic systems. ${ }^{1}$ Nevertheless, isotropic CAs self-assemble essentially in close-packed lattices, e.g. $f c c$ and $h c p$ structures, which are thermodynamically favored, as they gain more free volume entropy than other packing symmetries. For diversifying the achievable structures, anisotropic building blocks have been investigated in these last two decades. ${ }^{2-7}$ Among them, the concepts of colloidal molecules $(\mathrm{CMs})^{8,9}$ and more recently that of patchy colloids ${ }^{10-12}$ are particularly holding the researchers' interest, because their major merit is to offer directional and therefore limited colloidal interactions.

We aim with this review paper to highlight and classify the major contributions with a special focus on the most recent ones. We first describe the synthesis pathways to $\mathrm{CMs}$ recently enriched thanks to the science and technology of DNA. Then we introduce the interrelated concept of patchy colloids and present in a way as rational as possible the numerous and often highly inventive synthesis routes hitherto reported. Finally, we take stock of all the researchers' efforts to use patchy particles as building units to obtain new assemblies classified by increasing dimension, i.e. new discrete colloids (O-D), colloidal chains (1-D) and colloidal crystals (2-D and 3-D).

\section{Colloidal molecules}

The concept of CMs was first evoked by van Blaaderen in 2003 for describing non-spherical colloids stemming from the aggregation of a small number of particles. ${ }^{8,9}$ This perspective paper was in particular inspired by the pioneering work of Pine and co-workers. ${ }^{13}$ The latest developments of $\mathrm{CMs}$ were published and presented in several reviews.5,6,14-18 Among them, we published in 2011 an exhaustive critical review of the diverse synthesis routes for creating robust clusters of spherical particles which could mimic the space-filling models of exact simple molecules. ${ }^{15}$ For this occasion, we proposed a classification of CMs using and extending the well-known formalism of Gillespie derived from the Valence Shell Electron Pair Repulsion (VSEPR) model (Fig. 1). These routes deal with either the controlled clustering of preformed particles or strategies starting from a single particle, which is decorated by satellite ones by taking advantage on one hand of phase separation or on the other hand of nucleation and growth phenomena.

\subsection{Colloidal molecules through controlled clustering routes}

These routes start from preformed isotropic colloids whose clustering is assisted by depletion interaction, capillary condensation, van der Waals forces, electrostatic interactions, coalescence, chemical bonding, DNA hybridisation or 2-D and 3-D geometrical confinement. We deliberately omitted here those using patchy colloids that are described in the 5.1 section.

\footnotetext{
a. Univ. Bordeaux, CNRS, ICMCB, UMR 5026, F-33600, Pessac, France. E-mail:

etienne.duguet@icmcb.cnrs.fr

b. Univ. Bordeaux, CNRS, CRPP, UMR 5031, F-33600, Pessac, France
} 


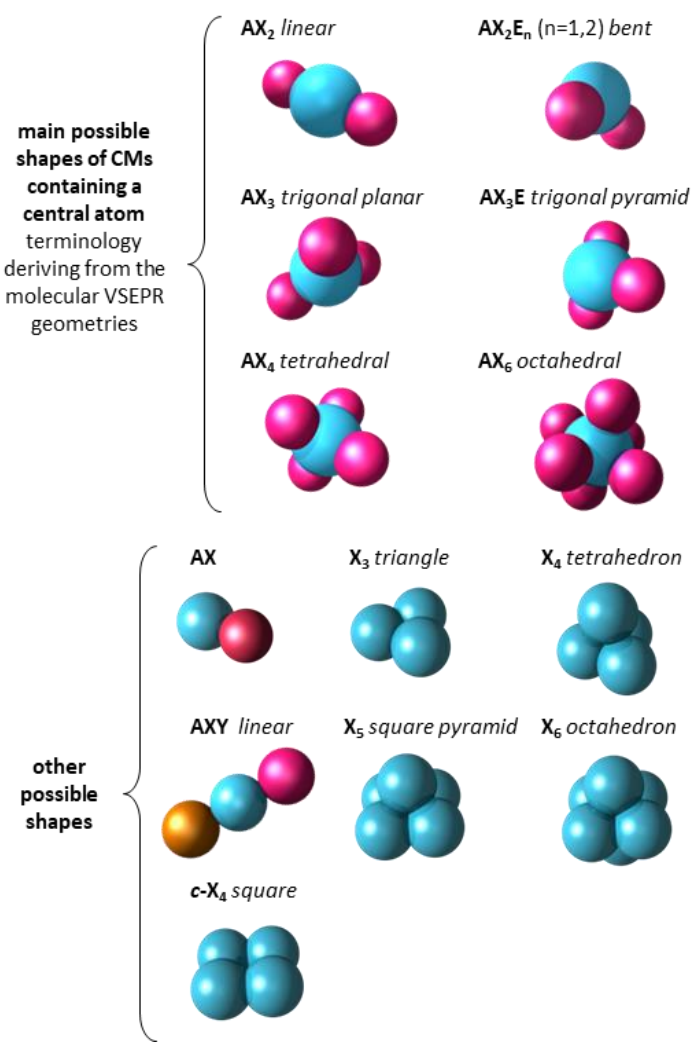

Figure 1 Extract of the classification of $\mathrm{CMs}$ as proposed earlier. Adapted from ref. ${ }^{15}$ with permission from the Royal Society of Chemistry.

Among the pioneering achievements, the work of Manoharan, Pine and co-workers is of particular interest concerning confinement within oil-in-water emulsion droplets containing polymer or silica microspheres. ${ }^{19,20}$ The subsequent controlled removal of the liquid from the droplets generates compressive forces that draw the particles together. When the spheres touch another on the surface of the droplets, removing more liquid causes the droplet to deform and generate capillary forces which collapse the particles into a cluster (Fig. 2)..$^{13}$ The structures of these clusters include familiar polyhedra such as tetrahedron $\left(\mathrm{X}_{4}\right)$ and octahedron $\left(\mathrm{X}_{6}\right)$, and ones that are more unusual. The selection of a unique packing arises almost entirely from geometrical constraints during the drying. ${ }^{21}$ Nevertheless, the size polydispersity of the initial emulsion droplet led to complex mixtures of different clusters that were finally sorted by density gradient centrifugation. In order to narrow the size distribution of the droplets, the emulsification stage was improved by shearing a polydisperse premix emulsion between two concentric cylinders and larger amounts of colloidal silica clusters with a linear, triangular and tetrahedral morphology were obtained. ${ }^{22}$ Later, Monte Carlo simulations allowed a full description of the dynamics correlated to experiments. ${ }^{23}$
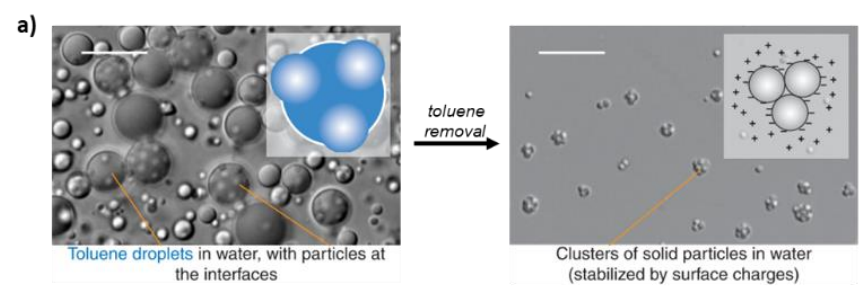

b)
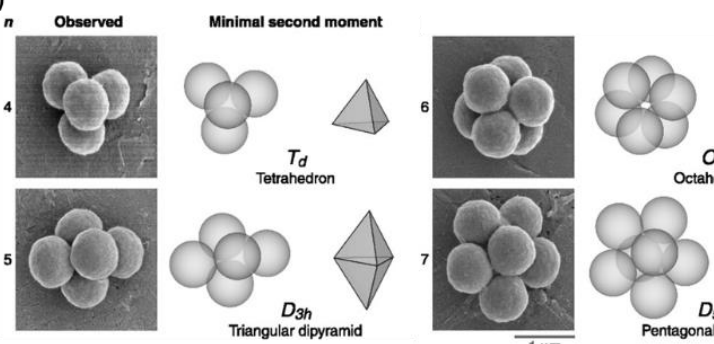

$O_{h}$

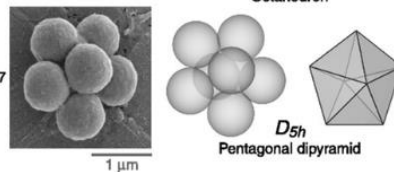

Figure 2 Clustering of polystyrene microparticles through their confinement within liquid droplet: a) optical micrographs and diagrams (insets) of the packing process and b) SEM images and geometry of some of the obtained CMs. Scale bars in a) and b) are 10 $\mu \mathrm{m}$ and $1 \mu \mathrm{m}$, respectively. Adapted with permission from ref. ${ }^{13}$ Copyright (c) 2003, American Association for the Advancement of Science.

Clustering can also result from the single protrusion emerging from the surface of crosslinked polymer microparticles when they are swollen with monomer and temperature is raised. In this way, Kraft et al. showed that delaying the polymerisation of the protrusions on polystyrene (PS) nanoparticles previously coated with vinyl acetate leads to coalescence of the droplets upon collision, thereby easily creating $\mathrm{CMs}$ that can be solidified upon polymerisation (Fig. 3). ${ }^{24,25} \mathrm{AX}$ to $\mathrm{AX} \mathrm{g}_{9}$ morphologies were obtained after solidification of the merged protrusions giving rise to the central CA. They were obtained as mixtures of different CMs types, but these were easily sorted by gradient density centrifugation. Interestingly, the swelling liquid can be an apolar solvent allowing to reconfigure any aggregate of PS spheres thanks to a diffusion-limited aggregation process. The swollen effect lowers the van der Walls forces and lubricates the contact area between the spheres, thereby yielding regular CMs through minimisation of the interfacial energy. For $A X_{6}$, octahedra coexist with polytetrahedra, which is strongly preferred over the minimal second-moment arrangement. The patch arrangement is uniform and random when the number of assembling particles is lower and more than 5, respectively. The final morphology can be tuned by varying the swelling ratio, swelling solvent, surfactant concentration, and swelling time. 


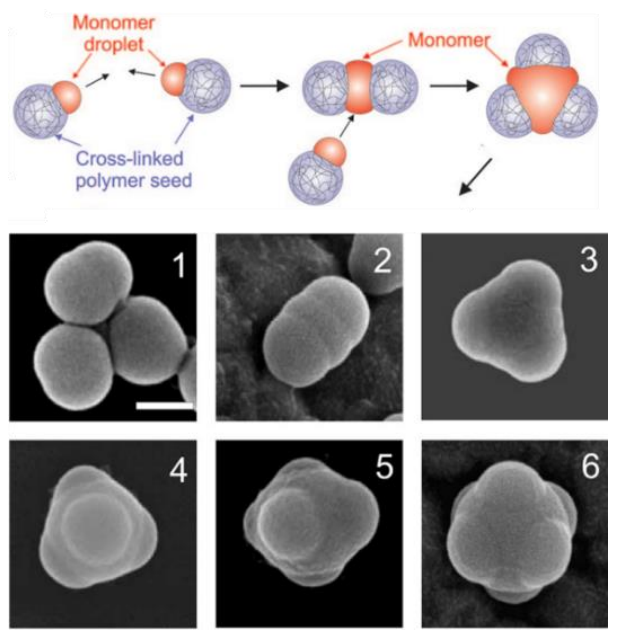

Figure 3 Schematic representation of merging of liquid protrusions yielding CMs and SEM images of $C M s$ from $A X$ to $A X_{6}$ types. Scale bar: $200 \mathrm{~nm}$. Adapted with permission from ref. ${ }^{24}$ Copyright $(2009$, American Chemical Society.

More recently, Zhang and co-workers reported a crystal templating strategy leading exclusively to $\mathrm{AX}_{12} \mathrm{CMs}$ from soft poly(N-isopropylacrylamide) (PNIPAM) microgel submicron spheres. ${ }^{26}$ When $f c c$ - or hcp-like colloidal crystals (CCs) are prepared from a 40:1 mixture of PNIPAM microspheres with surface thiol groups and surface vinyl groups, the latter are statistically in close contact with 12 microspheres bearing thiol groups. Under UV-light irradiation, the thiol-ene reaction allows binding covalently the 13 microspheres, which are recovered as independent clusters after the destruction of the CCs. Nevertheless, by-product CMs with a higher number of microspheres were found in the same batch as well when two vinyl-PNIPAM microgels were entrapped too closely, i.e. with less than two separating thiol-PNIPAM microgels. Nevertheless, they were easily separated by density gradient centrifugation. The same strategy was extended to $A X_{4}, A X_{5}$ and $A X_{6} C M s$ using vinyl-PNIPAM microspheres smaller than the thiol-PNIPAM ones in order to accommodate them in the interstitial sites of different close-packed lattices. ${ }^{27}$

According to a similar clustering strategy in confined 3-D environments, Tabeling and co-workers reported a strategy to obtain CMs $\left(X_{n}\right.$ with $n=3$ to 6$)$ in microfluidic channels. ${ }^{28}$ Closepacked planar $\mathrm{CMs}$ obtained under depletion conditions in a 2 D cell were studied by Manoharan and co-workers, ${ }^{29}$ while Grzybowski and coll. used 2-D virtual magnetic moulds. ${ }^{30}$

As demonstrated earlier by Alivisatos', Mirkin's and Sleiman's groups, DNA hybridization between complementary sequences anchored at the surface of particles provides a flexible tool to control the formation of $\mathrm{CMs}$ and in particular to assemble in a controled way colloids of different sizes or chemical compositions. ${ }^{31-34}$ Manoharan and co-workers showed that a yield as high as $90 \%$ is achievable for the fabrication of $\mathrm{AX}_{4}$ morphologies in the absence of confinement just by adjusting to 2.41 the size ratio between two sizemonodisperse populations of colloids. ${ }^{35}$ Indeed this is not specific to DNA driven assembly and the author demonstrated that electrostatic interactions between oppositely charged colloids yield similar results. Crocker and co-workers demonstrated that the interactions between DNA coated colloids can be reprogrammed using enzymes or reinforcing DNA strands which allow preparing $C M s\left(A X_{n}\right.$ with $n=4,6,8,10$ or 12) via an enhanced crystal templating method. ${ }^{36,37}$ For the enzymatic approach, two populations of DNA coated colloids are mixed, with a large excess of the larger ones, and forced into close-packed structures via centrifugation at low ionic strength. Sequence-specific enzymatic ligation at higher ionic strength creates a covalent bond selectively between colloids of different natures. A final washing step at low ionic strength destroys the crystals thereby releasing the covalently bound $\mathrm{CMs}$. The size ratio between the two populations of colloids determines the valence of the interstitial site occupied by the minority colloid and therefore the geometry of the final CM.

The most interesting use of DNA for CM design relies on DNA origamis, whose structure consists of a long single stranded plasmid folded by multiple smaller synthetic staples into arbitrary shapes. This design flexibility allows programming the assembly of DNA coated colloids with broad diversity of geometries. Gang and coworkers showed that directional and specific interactions between isotropic gold nanoparticles guided by DNA origamis allowed to form $X_{6}$, planar $c-X_{4}$ and planar $\mathrm{AX}_{4} \mathrm{E}_{2} \mathrm{CMs}$ in solution. ${ }^{38,39}$ In a key article on the design of CMs guided by DNA, Mao and co-workers used DNA cages, i.e. smaller DNA constructs than origami that consist only of synthetic strands. Trapping gold nanoparticles in these DNA cages can guides the assembly of $\mathrm{AX}_{4} \mathrm{CM}$ with yields as high as $67 \%$, and grants control over the geometry of $\mathrm{AX}_{6} \mathrm{CM}$ between octahedral (as in $\mathrm{SF}_{6}$ ) and trigonal prismatic (as in $\mathrm{W}\left(\mathrm{CH}_{3}\right)_{6}$ ) as well as more complex $\mathrm{CMs}$ such as dual-core ethane-like $\mathrm{CMs}$ frozen in an eclipsed conformation (Fig. 4). ${ }^{40}$

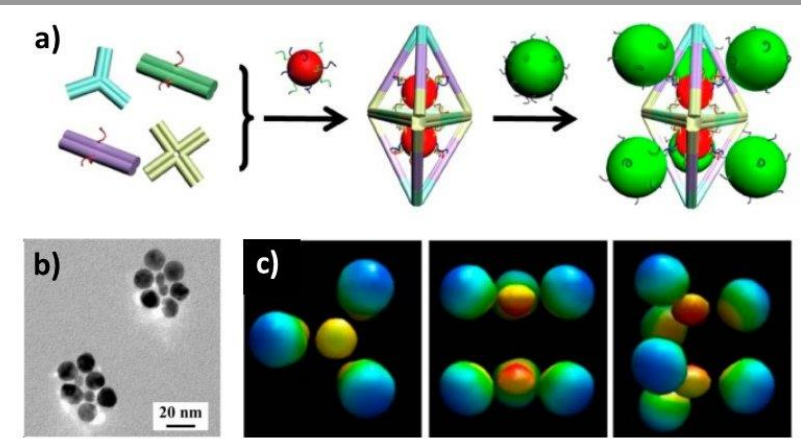

Figure 4 DNA frame guided-assembly of ethane-like CMs : a) schematic representation of the assembly guided by a DNA cage, b) the corresponding TEM image and c) different views of the 3D-reconstruction obtained by cryo electron microscopy. Adapted with permission from ref. ${ }^{40}$ Copyright (c) 2015, American Chemical Society.

A recurring problem with DNA-guided organisation of large colloids lies in the relative flexibility of the DNA architectures that fails to maintain the colloids in the desired geometry. To overcome this issue, Chaikin and co-workers designed a DNA origami belt that binds to the surface of a large colloid (about $700 \mathrm{~nm}$ ) and serves as guide for the later assembly of other DNA coated colloids. This strategy not only yields to chiral CMs on an $\mathrm{X}_{4}$ basis, but also allows controlling the dihedral angle between the particles (Fig. 5). ${ }^{41}$ 
a)

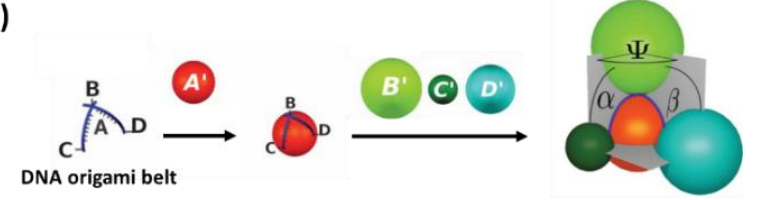

b) (R)
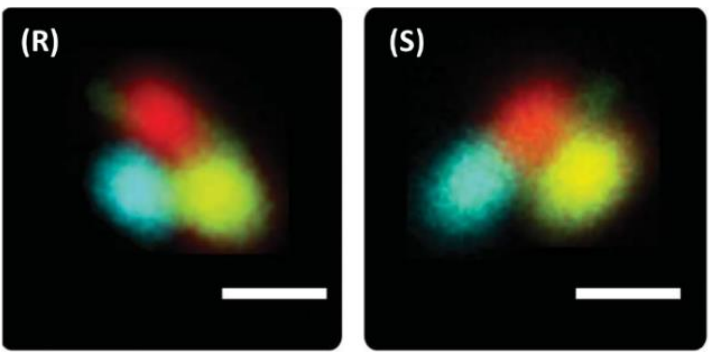

Figure 5 Formation of a chiral $\mathrm{CM}:$ a) schematic representation of the wrapping of a DNA origami belt around a first colloid which guides the assembly of 3 other colloids into a chiral $\mathrm{CM}$ with tuneable dihedral angles $(\alpha, \beta, \Psi)$ and b) confocal laser scanning microscopy images of two enantiomeric CMs. Scale bars: $1 \mu \mathrm{m}$. Adapted with permission from ref. ${ }^{41}$. Copyright @ 2017, American Association for the Advancement of Science.

The recent emergence of surface mobile DNA linkers provides new types of $A X_{n} C M s(n=2,3$ and 4$)$ with flexible joints, where the satellite colloids remain free to move around the central one. ${ }^{42}$ The colloids forming these structures consist of silica particles surrounded by a lipid-bilayer. DNA strands functionalized at their ends by cholesterol or stearyl moieties are anchored into this membrane. As long as the lipid bilayers remain fluid, the DNA strands are free to move around the particles and so are the joints formed between colloids with complementary strands at their surfaces. To a certain extent, this could mimic scissoring, rocking, twisting or wagging vibrations of conventional molecules.

Finally, the work of Wu and co-workers deserves also to be cited here because it made possible to obtain $C M s$ in 2-D confinement under an AC electric field which induces anisotropic interactions between negatively-charged PS microparticles and subsequent clustering. ${ }^{43}$ The authors observed different cluster morphologies, in particular of the $\mathrm{X}_{4^{-}}$ type, by varying the field frequency. They introduced later chirality by replacing microspheres by asymmetric microdumbbells and forming both right- and left-handed clusters on the conducting substrate. ${ }^{44}$ They further demonstrated that this broken symmetry induces an unbalanced electrohydrodynamic flow making them rotating in opposite directions according to their handedness. Nevertheless, strategies remain to be found for both fixing the structure of the CMs after suppression of the field and producing them in sufficient quantity to study their properties and possible applications. This emerging field of active colloidal molecules was recently reviewed by Löwen. ${ }^{45}$

\subsection{Colloidal molecules through controlled phase separation phenomena}

The more impressive achievements were obtained through seeded emulsion polymerisation. Emulsion polymerisation is a heterophase process of great industrial importance leading to stable colloidal dispersions of polymer particles in water, called "latex". ${ }^{46}$ Generally the polymer particles have final diameters in the range of 0.05-1 $\mu \mathrm{m}$. One of the critical features of emulsion polymerisation is the ability to control particle morphology, e.g. formation of core-shell particles and other equilibrium morphologies by successive additions of different monomers, i.e. seeded growth polymerisation. In particular, snowman-like morphologies can be obtained as the consequence of a phase separation phenomenon. The anisotropy was arisen from the immiscibility of the second stage polymer in crosslinked polymer networks, leading to the formation of a protrusion on one side of the seed latex particle. Overall, the phase separation process is favoured by increasing either the monomer/polymer swelling ratio, the seed crosslink density, the seed size, the temperature or the crosslinker concentration in the mixture of swelling monomers, etc. In some situations, when the seed polymer is more polar than the second stage polymer, e.g. poly(methyl methacrylate) (PMMA) and poly(n-butyl acrylate-co-styrene), and when the temperature of glass transition of the second stage polymer is less than the polymerisation temperature, several protrusions can be simultaneously obtained, but without control of their number, size and positions on the central seed latex. ${ }^{47}$

This seeded emulsion polymerisation route was revisited recently in order to prepare more complex latex microparticles combining polymers of different chemical compositions and/or more sophisticated shapes. ${ }^{48-50}$ Indeed, snowman-like colloids were designed by combining styrene, MMA, butyl methacrylate, etc. eventually copolymerised with a functional monomer, e.g. to make one of the nodules hydrophilic and therefore the whole colloid amphiphilic. ${ }^{48}$ Concerning shape variations, crosslinking density gradients were used to overcome the effect of surface tension and provide reproducible directionality to phase separations. Starting from snowman-like particles, i.e. dimers, whose both PS nodules were crosslinked at controlled densities, Weitz and coll. obtained linear $\left(\mathrm{AX}_{2}\right)$ or triangular $\left(\mathrm{X}_{3}\right)$ trimers. ${ }^{49}$ Indeed, the third nodules are capable of growing linearly from the less crosslinked nodules or in a perpendicular direction when the crosslinking densities of both nodules are similar (Figure 6a-c). Diamond particles $\left(c-\mathrm{X}_{4}\right)$ are made similarly to the triangle particles at the expense of using a $20 \%$ excess of crosslinker. Combining three swelling/polymerisation stages, Dufresne and co-workers fabricated CMs successfully with the symmetry of water molecules $\left(\mathrm{AX}_{2} \mathrm{E}_{2}\right)$ as shown in Fig. $6 \mathrm{~d} .{ }^{50}$ Interestingly, the bond angle can also be tuned from about 80 to $140^{\circ}$, vs $104.5^{\circ}$ within the true water molecule, by varying the swelling ratio in the final step. 

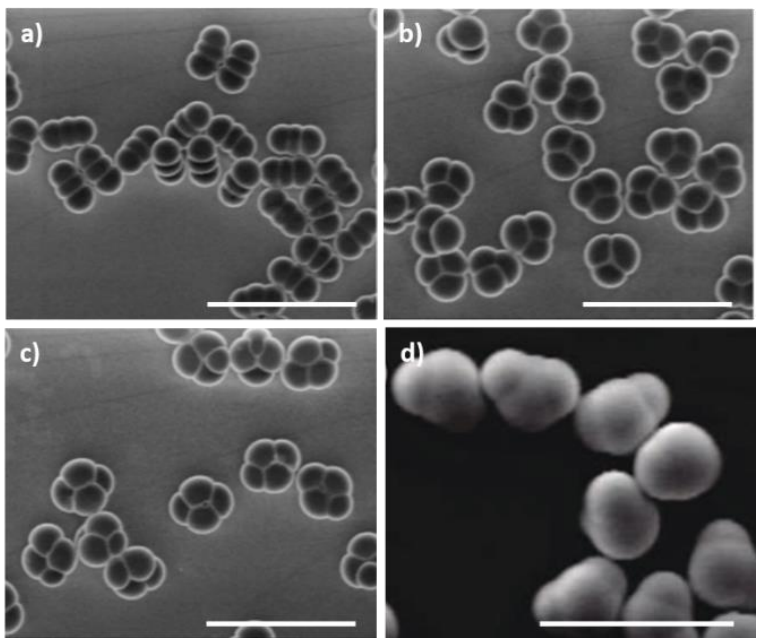

Figure 6 SEM images of a) $\mathrm{AX}_{2}$ dumbbell-like, b) $\mathrm{X}_{3}$ triangle-like and c) $\mathrm{cp}-\mathrm{X}_{4}$ diamondlike ${ }^{49}$ and d) $\mathrm{AX}_{2} \mathrm{E}_{2}$ water-like ${ }^{50}$ latex $C M$ s obtained through phase separation in seeded growth emulsion polymerisation. Scale bars: $20 \mu \mathrm{m}$. Adapted with permission from ref. ${ }^{49}$ Copyright (C) 2007 WILEY - VCH Verlag GmbH \& Co. KGaA, Weinheim. Adapted with permission from ref. ${ }^{50}$ Copyright $(C) 2009$, American Chemical Society.

More recently, Peng, Imhof and coll. reported that $\mathrm{AX}_{2} \mathrm{CMs}$ can be obtained in one stage from a conventional spherical 3- $\mu \mathrm{m}$ latex seed made of crosslinked PMMA. ${ }^{51}$ They showed by realtime optical microscopy that a large number of small droplets of swelling monomers form simultaneously on the surface of the seeds, and then fuse until two protrusions remaining on the opposite sides. Stirring not only accelerates the swelling process but also induces the coalescence of the liquid protrusions from different seeds leading to $C M s$ with $A_{2} E_{2}, A X_{3} E$ and even acetylene-like morphologies. The subsequent polymerisation makes the structures permanent.

Lastly, more advanced $A X_{3} E$ and $A X_{2} E_{2} C M s$ made of $P S$ for the central latex particle and poly(4-vinylpyridine) (P4VP) for the satellites were reported by Wang and coll. ${ }^{52}$ Accordingly to the pioneering studies, the phase separation occurs simultaneously to the polymerisation of the swelling monomer and not before as shown in the just above work.

\subsection{Colloidal molecules through controlled surface nucleation and growth}

This synthesis pathway leads essentially to $A X_{n}$-type $C M s$ and consists in the controlled nucleation and growth of the satellite colloids onto the surface of the central one, which plays the role of a preformed seed. Contrarily to the previous section, the seed cannot be swollen by the monomer, because this is generally an inorganic nanoparticle. Seeded growth emulsion polymerisation is well-suited to obtain hybrid CMs in size range of few hundreds of nanometres while dispersion polymerisation leads to larger CMs but generally with less good control of the morphologies. ${ }^{15}$

We reported an efficient recipe where a controlled number of PS latex nodules grow onto the surface of silica seed particles. ${ }^{53,54,55}$ The surface of these silica seeds needs to be previously treated by compatibilizers, e.g. methacryloxyalkyltrimethoxysilane at low surface density, in order to create reactive (co)polymerisable loci promoting the surface capture of the growing PS macromolecules and therefore the nucleation of the latex particles. In the range of 0.1-1 molecules per $\mathrm{nm}^{2}$, we showed that the higher the silane surface density, the more encapsulating the PS nodules. Original cryo-electron tomography experiments allowed to follow-up the nucleation and growth of the PS nodules. ${ }^{56}$ Early samples showed the presence of numerous small PS nodules bound to silica seeds in a random distribution. As far as the polymerisation is running, the number of PS nodules per silica seed decreases, leading to morphologies imposed by the satellite steric repulsion, i.e. by the satellite-to-seed size ratio. This ratio can be tuned through experimental parameters such as the seed concentration, seed diameter, monomer concentration, and fraction of the sodium dodecylsulfate introduced as co-surfactant as a complement to the main surfactant which is the polyethylene glycol nonylphenyl ether. A model was proposed to help understand the mechanism of the nodules growth ${ }^{57}$ and predict the optimal recipe parameters for a targeted morphology. ${ }^{58}$ This comprehensive study allowed to produce an extensive range of CMs and to reach morphology yields higher than $80 \%$, in particular for the $\mathrm{CMs}$ deriving from Platonic solids, e.g. $A X_{4}, A X_{6}$ and $A X_{12}$, which correspond to tetrahedra, octahedra and icosahedra, respectively. $A X_{3}, A X_{8}$ and $\mathrm{AX}_{9} \mathrm{CMs}$ were also obtained. $\mathrm{AX}$ and $\mathrm{AX}_{2}$ morphologies were achieved when the surface density and hydrophobicity was adjusted, i.e. replacing methacryloxymethyltrimethoxysilane (MMS) by methacryloxypropyltrimethoxysilane (MPS) (Fig. 7a). ${ }^{59}$

A recent study showed that quite similar $\mathrm{CMs}$ are achievable when using a different mechanism combining nitroxidemediated polymerisation and induced self-assembly of amphiphilic block copolymers, but the morphology yields were not determined. 60

Lastly, the AXY triphasic CMs with a silica core, one PS nodule and one PMMA nodule were obtained through a twostage protocol, where a PS nodule was conventionally created in a first step. ${ }^{61}$ To forbid the polymerisation of the MMA molecules possibly swelling the PS nodule and promote successfully the nucleation of the MMA nodule on the other seed side, the silica surface was previously treated by a cationic initiator, namely 2,2'-azobis $\left(\mathrm{N}, \mathrm{N}^{\prime}\right.$-dimethylene isobutyramidine) dihydrochloride (Fig. 7b). 
a)

a)

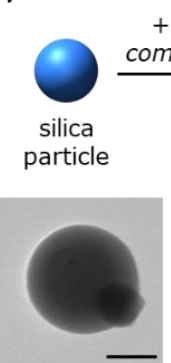

AX ilica

$$
\text { + surface }
$$
compatibilizer

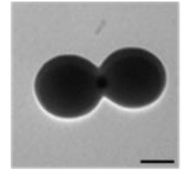

$\mathrm{AX}_{2}$

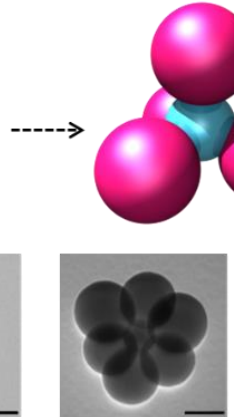

$\mathrm{AX}_{6}$ b)

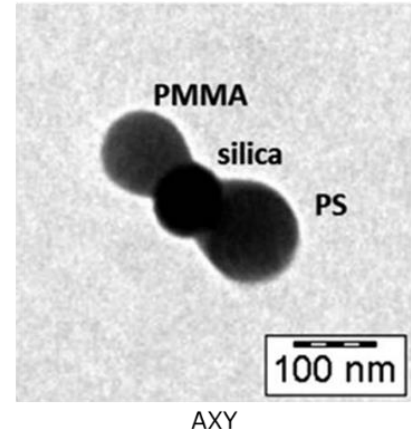

Figure 7 a) silica/PS $A X_{n}$-type CMs obtained through seeded growth emulsion polymerisation of styrene in the presence of MMS-modified silica seeds: synthesis scheme and TEM images of the as-obtained CMs; ${ }^{59}$ b) TEM image of the AXY-type CM made from a silica seed with one PS nodule and one PMMA nodule. ${ }^{61}$ Scale bars: 100 nm. Adapted from ref. ${ }^{59,61}$ with permission from the Royal Society of Chemistry.

\section{Colloidal molecules vs patchy colloids}

When van Blaaderen introduced the concept of CMs, he took into account not only that spherical colloids can be treated as if they were atoms, but also that molecules form more complex materials than atoms do. ${ }^{8,9} \mathrm{~A}$ pioneering work by Dufresne and co-workers consisted in assembling $\mathrm{X}_{2}$ CMs obtained by phase separation in seeded emulsion polymerisation. ${ }^{62}$ They used an external electric field to align and assemble the PS-based dumbbells (with a diameter of $270 \mathrm{~nm}$ and an overall length of $420 \mathrm{~nm}$ ) to make a birefringent suspension with structural colour that disappears when the electric field is turned off. When the solvent was removed in the presence of the electric field, the particles self-assemble into a CC hundreds of particles thick with a record packing fraction of 0.7862 . The probably most exciting result was recently reported by $\mathrm{Yi}$, Pine and coworkers and consisted in co-assembling 780-nm PS spheres with $\mathrm{X}_{4} \mathrm{CMs}$ made of four 650-nm PS spheres. ${ }^{63}$ Each type of building blocks is previously made fluorescent and surface modified with complementary ss-DNA strands, which creates a temperature-dependent attractive interaction between the $\mathrm{CMs}$ and the spheres. Helped by weak depletion conditions, crystallisation occurs successfully when decreasing very slowly the temperature near the aggregation temperature leading, as expected from simulations, to colloidal isomorphs of the $\mathrm{MgCu}_{2}$ Laves phase, i.e. a diamond lattice that interpenetrates a pyrochlore lattice (Figure 8). a)

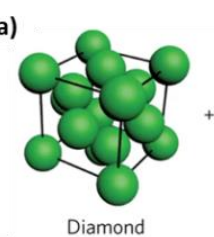

b)

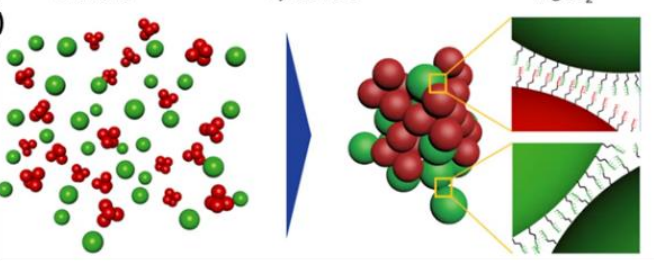

c)

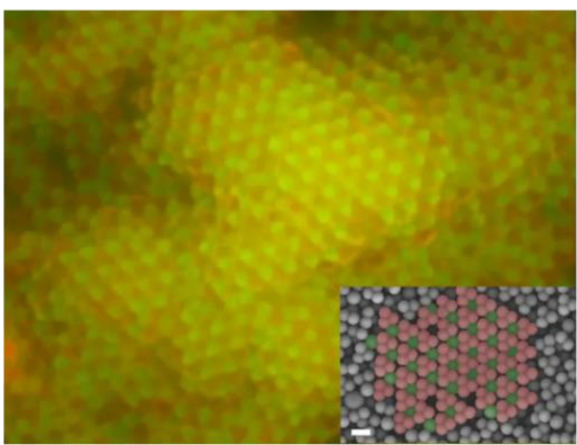

Figure 8 a) the $\mathrm{MgCu}_{2}$ superlattice made up of two sublattices, diamond in green and pyrochlore in red; b) the mixture of PS spheres (in green) and $\mathrm{X}_{4} \mathrm{CMs}$ (in red) expected to interact through complementary surface ss-DNA strands; $c$ ) fluorescence microscope image of system-spanning crystals with the $\mathrm{MgCu}_{2}$ structure formed after slow cooling through the melting temperature (the field of view is $50 \mu \mathrm{m} \times 50 \mu \mathrm{m}$ ). Inset: an electron microscope photograph of a section of the $\mathrm{MgCu}_{2}$ crystal whose different building blocks is evidenced with false colours. Scale bar: $1 \mu \mathrm{m}$. Adapted with permission from ref. ${ }^{63}$ Copyright (c) 2017, Springer Nature.

However, 16 years later after the birth of the CM concept, it must be admitted that these examples are surprisingly very few. This is probably due not only to the lack of morphological purity of most of the batches of CMs obtained - thus prohibiting any possibility of crystallization on a large scale -, but also to the fact that the researchers focused more on controlling their geometry, at least initially, rather than on their assembly capability. A third reason is that the concept of patchy particles has made its way in the meantime. ${ }^{10-12}$ These are patterned 
particles with chemical or topological surface discontinuities which are expected to promote assembly in a limited number of specific directions. These predetermined instructions for directional assembly can be related to the atomic concept of valence extended here at the colloidal scale. That is why patchy particles are also sometimes called patchy colloidal atoms or valence-endowed colloidal atoms. When the patch is "chemically sticky", it is called enthalpic patch. When it corresponds to topological patterning, e.g. surface dimples, allowing for locking another particle with a complementary shape in depletion conditions, this is called entropic patchiness.

Both concepts of $\mathrm{CMs}$ and patchy colloids are closely related: firstly, because some types of $\mathrm{CMs}$ can be the precursors of patchy particles; secondly, because patchy particles can be original building blocks for new CMs, among other assembly types. The details will be given in sections 4.2.2 and 5.1.1, respectively.

The concept of patchy particles can also be seen as an extension of that of Janus-like particles. In the field of science, the particle consisting of two faces with different properties is called as Janus particle by de Gennes ${ }^{64}$, which is quite close to the connotation of Yin-Yang (阴-阳) in Chinese philosophy illustrating the interconnection between the seemingly opposite and/or contrary forces in the natural world. These Janus particles have various applications, such as giant surfactant ${ }^{65,66}$, micromotors ${ }^{67}$, etc. If one hemisphere is attracting while the second one is repulsing, Janus-like particles can be seen as one-patch particles. Patchy particles, i.e. particles with at least two patches, are less easy to be fabricated all the more so as the variability can concern not only the patch size and the interaction type but also the number, shape and relative position of the patches (Figure 9a). ${ }^{68}$ Moreover, the perspective of enthalpic and/or entropic patchiness leads to consider also the flat, concave or convex shape of the patches (Figure 9b).

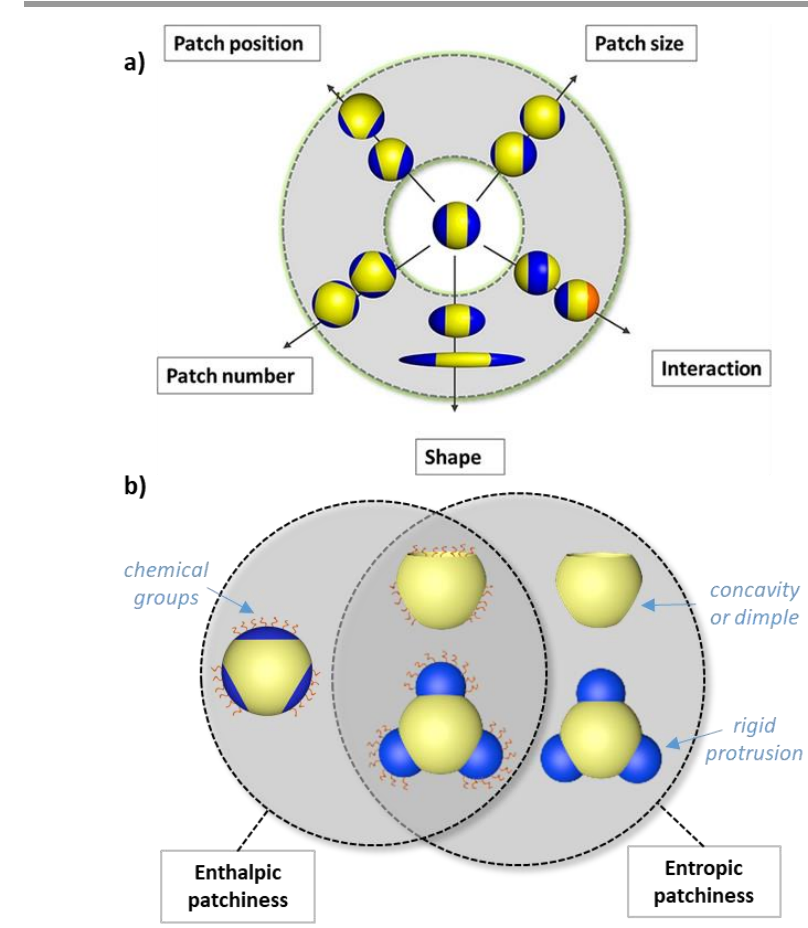

Figure 9 Schematic showing a) the various parameters to be tuned for controlling the assembly capability of patchy particles ${ }^{68}$ and b) the differences and interrelations between enthalpic and entropic patchiness. Reproduced with permission from ref. ${ }^{68}$ Copyright @ 2012, American Chemical Society.

\section{Synthesis routes of patchy particles}

Several techniques have been investigated and optimised to fabricate patchy particles. Among them, some are specific to a particular number of patches, while others are more versatile. This state-of-the-art is restricted to the synthesis routes leading efficiently to the creation of a maximum number of twelve patches regularly arranged all around the particle, describing repulsion figures similar to those of the VSEPR model. In other words, the raspberry-like structures have deliberately been omitted.

\subsection{Strategies specific to a particular number of patches}

\subsubsection{One-patch particles}

As previously mentioned, one-patch particles can be seen as Janus-like particles whose one of both hemispheres is or could be made attractive or sticky. Their synthesis was intensively investigated these last three decades and reviewed many times. ${ }^{69-74}$ It would be too long to present here the numerous synthesis pathways that allow breaking the symmetry of isotropic colloids. We focus on those which can also be combined with other techniques to obtain a higher number of patches or which led to building blocks useful for self-assembly purpose.

Briefly, particles to be made dissymmetric are partially trapped into or contacted on a flat/curved interface and the exposed side is regioselectively functionalized to create the patch. When the substrate is solid and the reagents are gaseous, the particles must be previously spread densely on the solid surface through spin coating ${ }^{75}$, evaporation ${ }^{76}$, etc. 
Kretzschmar and Pawar developed the glancing angle deposition (GLAD) method to partially coat with a metal or metal oxide a close-packed monolayer of particles, whose neighbouring particles and the substrate serve as protecting masks. ${ }^{77}$ The position, shape and coverage extent of the patch can be controlled by the orientation angle $\alpha$ of the layer and the deposition angle $\theta$ (Figure 10). a)

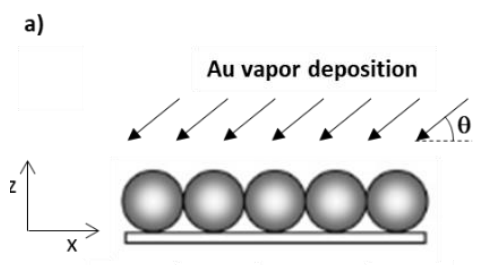

b)

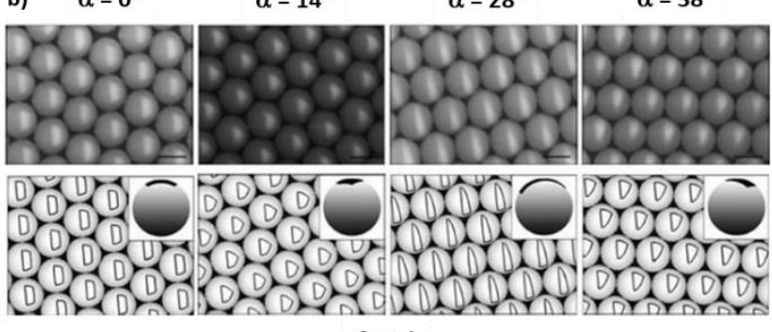

$\theta=2^{\circ}$

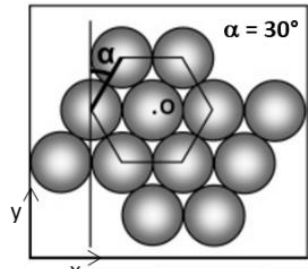

$\alpha=28^{\circ} \quad \alpha=38^{\circ}$
Figure 10 GLAD technique: a) schematic describing the process and evidencing both angular parameters and $b$ ) top row: SEM images of gold patches on 2.4- $\mu \mathrm{m}$ PS particles prepared by tuning the incidence angle and monolayer orientation, and bottom row: numerical simulation of patches at given angles. Scale bars: $2 \mu \mathrm{m}$. Adapted with permission from ref. ${ }^{77}$ Copyright $(C) 2008$, American Chemical Society.

The dissymmetrisation interface can also be solid-liquid such as wax-in-water emulsions, as reported first by Moore, Granick and coll. ${ }^{78}$ The Pickering emulsion is achieved by the stabilisation of the particles within the temperature range of the liquid wax. After solidification of the wax droplets, the exposed surface is regioselectively functionalised 79,80 or partially etched to form shape-anisotropic particles. ${ }^{81}$ The wax Pickering strategy allows tuning the patch extent through the control of the trapping degree of the particle into the wax phase by playing with the ionic strength, $\mathrm{pH}$ value, etc.

Concerning the preparation of one-dimple polymer particles, Sacanna and coworkers first reported a synthesis based on size-monodisperse silicon oil droplets nucleated via a base-catalyzed hydrolysis and condensation of 3(trimethoxysilyl)propyl methacrylate (TPM, also named MPS by other authors). ${ }^{82}$ These droplets later deform to reproducible shapes via a polymerization-induced buckling instability triggered by the thermal decomposition of a water-soluble radical initiator, e.g. potassium persulphate. Then Yi and coll. reported another efficient route based on a phase separation phenomenon occurring during a temperature-controlled swelling-deswelling process. ${ }^{83}$ The conventional PS latex particles are firstly swollen by decane, which becomes partially soluble in the water-ethanol medium at $60^{\circ} \mathrm{C}$; then the phase separation happens when decreasing the temperature (Fig. 11). The following removal of surfactant and the evaporation of the oil phase lead to particles with one hydrophobic dimple while the rest of the surface remains hydrophilic due to the presence of the surfactant.

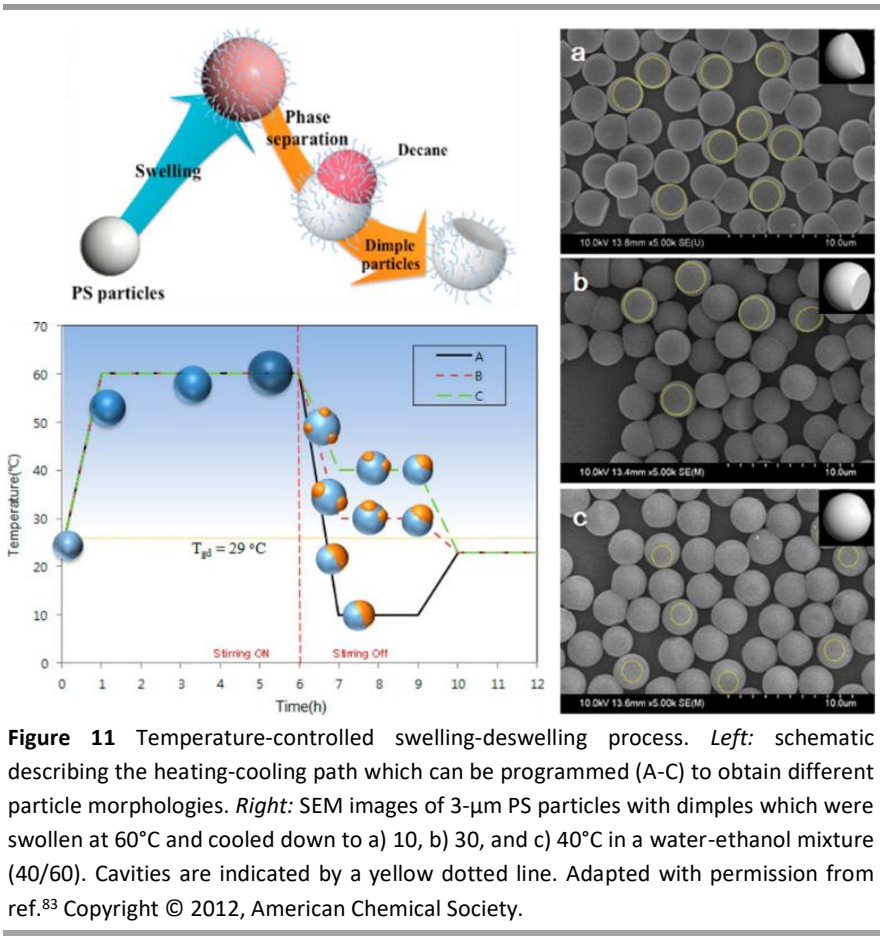

\subsubsection{Two-patch particles}

The creation of two patches oppositely positioned on an isotropic spherical particle necessitates often two stages. The GLAD method can be used twice if a particle stamping technique is employed using a polydimethylsiloxane stamp to invert the close-packed particle monolayer making the patches from the first vapour deposition facing down (Fig. 12). ${ }^{84}$ The accuracy of the stamping technique lies in efficiently inverting the monolayer and this was evaluated by comparing the experimentally observed patch geometries of the two-pole patchy particles to the mathematically predicted ones. The patch complexity can also be increased by tuning the template orientation and the beam incident angle simultaneously. ${ }^{85}$

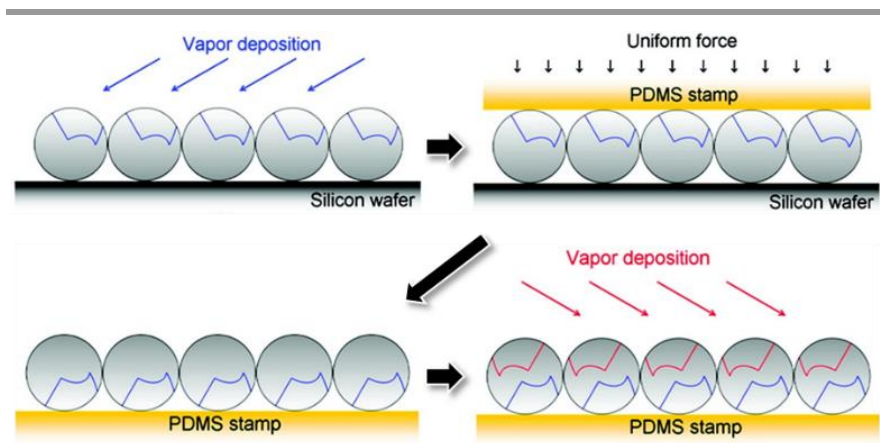

Figure 12 Cross-sectional schematic of the GLAD and particle stamping techniques to obtain two-pole patchy particles. Adapted with permission from ref. ${ }^{84}$ Copyright (C) 2009, American Chemical Society.

Lastly, crystalline particles present the advantage of exposing facets of different atomic densities and different affinities for adsorbing or reacting molecules accordingly. Kumacheva and 
co-workers took advantage of the presence of $\{111\}$ facets specifically at both ends of gold nanorods to create two opposite patches. ${ }^{86}$ Cetyltrimethylammonium bromide (CTAB) is well-known to be an efficient structuring agent for gold nanorods through the seeded growth method considering the larger affinity of CTAB to the $\{110\}$ facet on the body part. ${ }^{87}$ Then, at the ends of the nanorod, CTAB can be more easily shifted from the $\{111\}$ facets for instance by thiol-ended PS macromolecules leading to two hydrophobic patches capable of chaining the nanorods. ${ }^{86}$

\subsubsection{Six-patch particles}

In the same vein, Yi and co-workers created successfully six silica patches from the six faces of hematite nanocubes. ${ }^{88}$ In their presence, water droplets nucleate on the surface of hematite by phase separation in pentanol. By adjusting the water concentration, six droplets can form on each face of the hematite cube. When tetraethoxysilane (TEOS) is then added, it gradually diffuses into the water droplets through the oil phase. Within the droplets, hydrolysis and condensation of the precursors take place, leading to the formation of silica rods. In order to obtain flat silica patches, a small amount (1\%) of hydrophobic glycidoxypropyl trimethoxysilane (GPTMS) is added in the precursor to reduce the contact angle of water droplets on silica. TEOS is consumed at the initial stage forming a small neck, and then GPTMS is consumed to form large plates (Fig. 13).

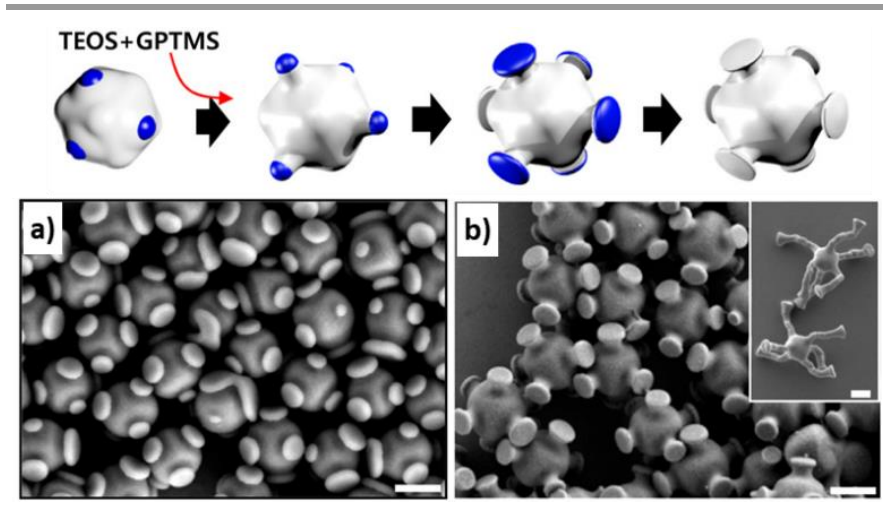

Figure 13 Left: schematic illustration of the formation of six silica patches on hematite cubes in pentanol. The blue dots represent the water droplets. Right: SEM images of the as-obtained six-patch particles from hematite cubes (edge length: $950 \mathrm{~nm}$ ) at a) $5^{\circ} \mathrm{C}$ and b) $10^{\circ} \mathrm{C}$. Inset shows longer tack-like patches grown at room temperature. Scale bars: 1 $\mu \mathrm{m}$. Adapted with permission from ref. ${ }^{88}$ Copyright (c 2018, American Chemical Society.

\subsubsection{Twelve-patch particles}

Twelve corresponds to the coordination number of spherical size-monodisperse particles in close-packed CCs. According to a strategy similar to the 3-D geometrical confinement in CCs (cf. 2.1 section), Zhao and co-workers prepared opals from 415-nm silica spheres and annealed them at $750^{\circ} \mathrm{C}$ in order to allow connections between adjacent spheres. ${ }^{89}$ Subsequently, the CC was immersed in a solution containing MPS to functionalize the exposed surface of the particles. Washing and redispersion yielded individual particles, whose surface was patterned by MPS (the connection areas were not modified by MPS). To reveal the patchiness, the authors used them as seeds in a subsequent regrowth of silica. Silica was successfully condensed only on the unmodified areas giving rise to twelve silica protrusions whose diameter can be controlled by the connection size, i.e. the annealing time. Nevertheless, a fraction of the particles had a silica nodule number different from twelve: not only those which were not fully coordinated because located on the surface or edges of the CCs, but also those inside the CCs that were not fully coordinated for sizepolydispersity reasons, i.e. too large distance between some particles to achieve the connections. More recently, Bae et al. developed a similar strategy but used octadecyltrichlorosilane self-assembly monolayer preferentially to MPS for growing titania patches. ${ }^{90}$ They compared ultrasonic and conventional agitation during patch growth, and they found that enhanced mass transfer is the key to controlled, homogeneous transport of the molecular precursors in a solvent onto the nanoparticles.

\subsection{Versatile strategies}

\subsubsection{Template-free methods}

Klupp Taylor and co-workers reported a one-pot approach to patchy particles based on the heterogeneous nucleation of silver onto the bare surface of calcined silica followed by surface diffusion dominated growth. ${ }^{91}$ The method was extended to the formation of gold patches onto cationic PS nanospheres using ascorbic acid to reduce the auric ion. ${ }^{92}$ Among several other experimental parameters, $\mathrm{pH}$ value and ascorbic acid concentration appeared to be critical parameters to control the patch morphology and number. Nevertheless, this technique is quite far to allow fine control of the size and relative position of the patches.

Bon and coll. reported that when using polymer nanogels as stabilisers in emulsion polymerisation of styrene, surface segregation of the nanogels yielded to the formation of patches whose number and size can be tuned by controlling the initial number of nanogels, ionic strength and $\mathrm{pH}$ value (Fig. 14). ${ }^{93}$ Once again, the control of the relative positions of the patches was not demonstrated.

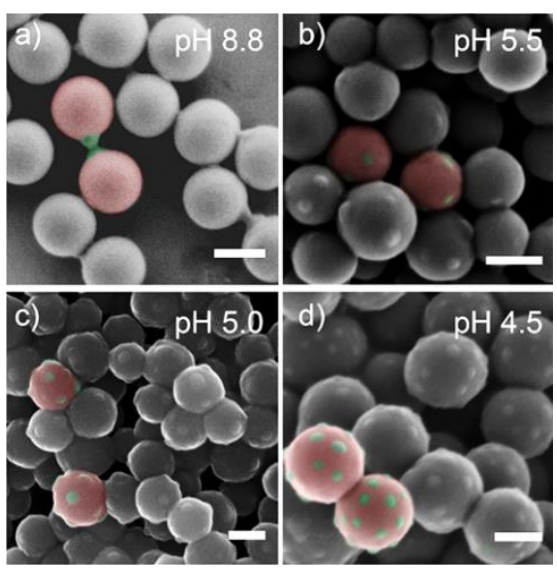

Figure 14 False coloured SEM images of PS latex particles obtained by emulsion polymerisation stabilised by nanogels showing that the number of patches made of nanogels aggregates depends on the $\mathrm{pH}$ value prior to polymerisation. Scale bars: 100 $\mathrm{nm}$. Reproduced with permission from ref..$^{93}$ Copyright (C) 2019, American Chemical Society. 


\subsubsection{Derivatisation of preformed colloidal molecules}

As previously discussed, CMs and patchy particles are quite close concepts. In particular, CMs whose satellites particles are naturally sticky or could be made sticky under external stimuli such as temperature, solvent, etc. can also be considered as patchy particles. However, CMs are more often potential precursors of patchy particles subject to some chemical modifications.

In this way, the micron-sized CMs obtained by Pine, Weck and coll. through confinement in liquid droplets were derivatised in two types of patchy particles. Patchy particles with DNA functionalised patches were obtained from CMs made of amidinated PS microspheres. ${ }^{94}$ They were swollen by styrene in order to let emerging amidine patches. After styrene polymerisation, biotin was regioselectively grafted on the patches, prior to the anchoring of biotinated DNA oligomers via a biotin-streptavidin-biotin linkage (Fig. 15 left). Particles with concave patches, i.e. dimples, were created differently from silica CMs, partial encapsulation in TPM which was then polymerised before silica etching with fluorhydric acid (Fig. 15 right). ${ }^{95}$ These strategies were successfully implemented to a quite large series of CMs. Thanks to a modified version of the protocol, TPM-based particles were obtained with azide moieties on the surface of the convex patches and carboxylic acids on the shell. ${ }^{96}$ Strain-promoted alkyne-azide cycloaddition and carbodiimide-mediated amidation were the two orthogonal coupling strategies used to anchor single-stranded oligonucleotides with distinct terminal sequences. A mixed strategy combining PS CMs and TPM also allowed to obtain particles with concave or convex features on demand. ${ }^{97}$ The design is based on the solvent-induced shifting of the patch shape. The initial concave patchy particles were synthesised in a water suspension by a swelling-induced buckling process. Upon exposure to different solvents, the patches were tuned reversibly to be either concave or convex. Lastly, Wang and coworkers showed that promoting a dewetting step by adding a surfactant between the cluster partial encapsulation in the TPM droplet and its polymerisation allows to lower the symmetry of the $\mathrm{CMs}$, e.g. from $\mathrm{AX}_{2}$ to $\mathrm{AX}_{2} \mathrm{E}_{2}$ or from $\mathrm{AX}_{3}$ to $\mathrm{AX}_{3} \mathrm{E}^{98}$

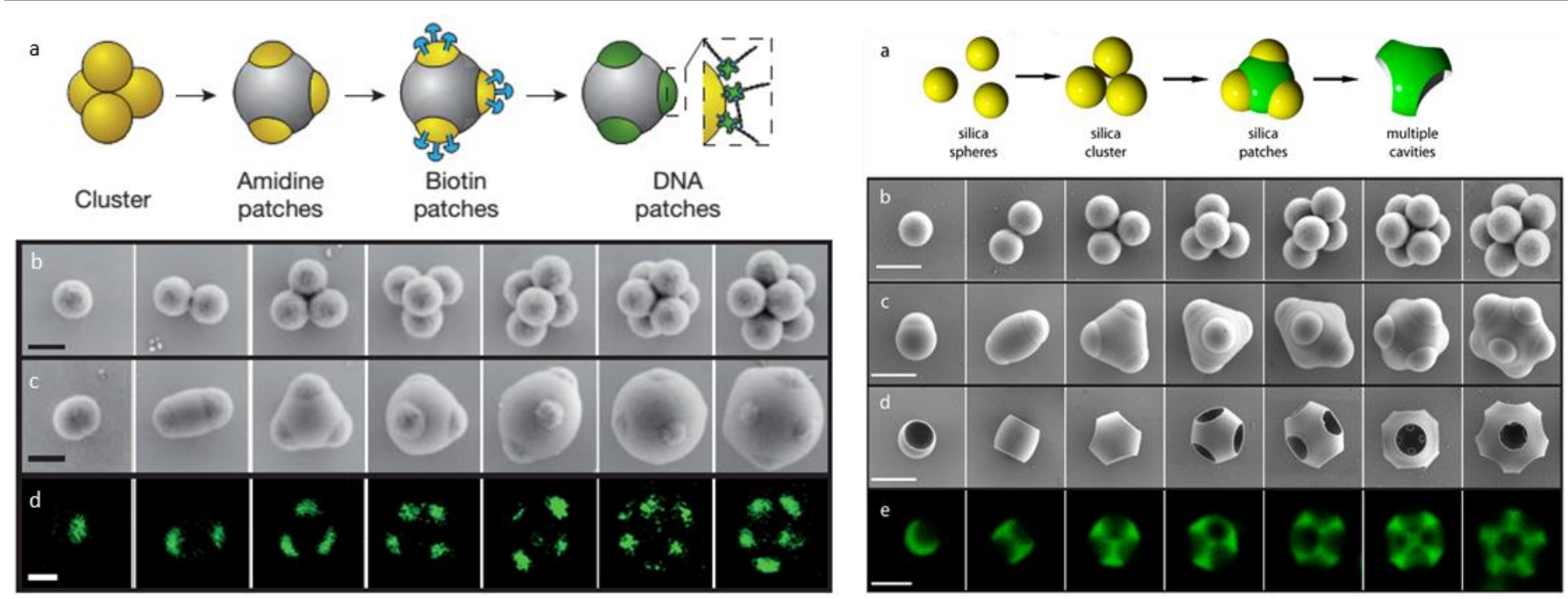

Figure 15 Left: fabrication of DNA-based patchy PS particles. a) Synthesis route. SEM images of amidinated, b) PS CMs and c) patchy particles after encapsulation. d) Confocal fluorescent images of the latter. Scale bars: $500 \mathrm{~nm}$. Reproduced with permission from ref. ${ }^{94}$ Copyright (C) 2012, Springer Nature. Right: fabrication of dimpled particles. a) Synthesis route. Electron micrographs of b) silica CMs, c) silica-TPM patchy particles, and d) multicavity particles. e) Confocal fluorescent images of fluorescently labelled multicavity particles. Scale bars: $1 \mu \mathrm{m}$. Reproduced with permission from ref. ${ }^{95}$ Copyright @ 2014, American Chemical Society.

An even more innovative strategy was carried out by Sacanna and coll., who envisioned and implemented the concept of colloidal fusion. ${ }^{99}$ Using coordination dynamics and wetting forces, it consists in parking PS particles on a liquid core droplet made of curable silicone oil to obtain hybrid liquid-solid clusters that evolve on the addition of a plasticiser to spherical particles with liquid surface patches capable of forming curable bonds with neighbouring particles (Fig. 16). This method provides high morphological yields and could $a$ priori be up-scaled.
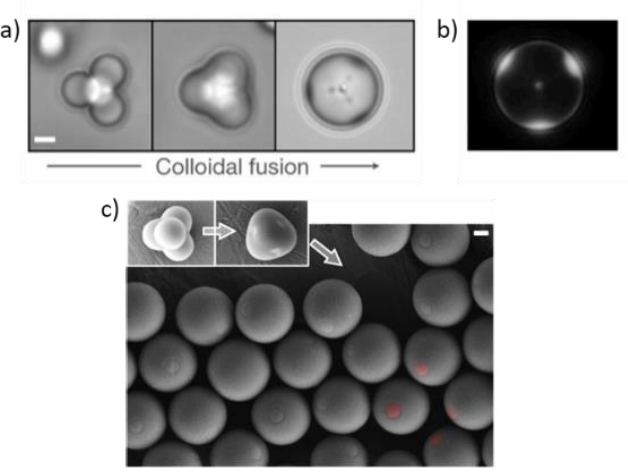

Figure 16 Colloidal fusion: a) bright-field time-lapse images showing the deformation of a tetrahedral liquid-core colloidal cluster after the addition of the plasticiser; b) fluorescence microscopy image at the final time point; c) SEM image of polymerised 
patchy particles (some patches are false-coloured). The insets show SEM images of deformed clusters with increasing degrees of plasticisation. Scale bars: $1 \mu \mathrm{m}$. Adapted with permission from ref..$^{99}$ Copyright (c) 2017, Springer Nature.

The silica/PS CMs obtained in our group by controlled surface nucleation and growth were also successfully converted into silica colloids combining entropic and enthalpic patchiness. ${ }^{59,100}$ Those were easily obtained by first regrowing the silica core and then removing the PS nodules by simple dissolution (Fig. 17). Not only the as-created dimples present the same features than those of the template satellites in terms of number, size and position, but also their bottom is covered by PS macromolecules originating from the copolymerisation of some growing PS chains with the surface-grafted MPS compatibilizer. These macromolecules make the dimples naturally sticky via hydrophobic interactions, but they can also be derivatised to developed ionic interactions or covalent bonding.

a)

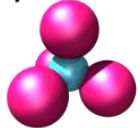
$\mathrm{AX}_{4}$-like $\mathrm{PS} /$ silica CM
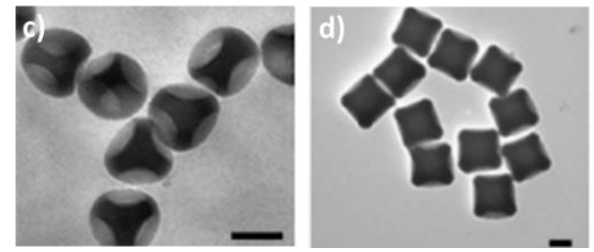

PS CMs into silica particles with pat enthalpic and entropic: a) synthesis route; b) STEM-EDX mapping evidencing the presence of PS residue at the bottom of the dimples; TEM images of patchy silica particles so-obtained from silica/PS CMs with c) four, d) six and e) twelve PS nodules. Scale bars: $100 \mathrm{~nm}$. Adapted with permission from ref. ${ }^{100}$ Copyright $@ ~ 2013$, WILEY-VCH Verlag GmbH \& Co. KGaA, Weinheim.

\subsubsection{Phase separation and reshaping}

We discussed in the 1.1.2 section how controlled phase separation phenomena could be exploited to generate protrusions in the course of emulsion polymerisation seeded with polymer latex particles. This method was also used by Wang, Song and coll. to fabricate dimpled particles by controlling the adding speed of crosslinker at different stages (Fig. 18). ${ }^{101}$ The formation of mono- or multi-cavities is attributed to the single- or multi-location of polydivinylbenzene (PDVB) at the latex surface. Specifically, DVB added at slow speed prefers to locate at one place and induces the single cavity after phase separation to reduce Gibbs interfacial free energy, as the network of PDVB is more rigid than the core seed. However, multi-cavity particles can be fabricated alternatively if DVB is fast added. Nevertheless, it was not demonstrated that the number of cavities could be finely tuned through this strategy.

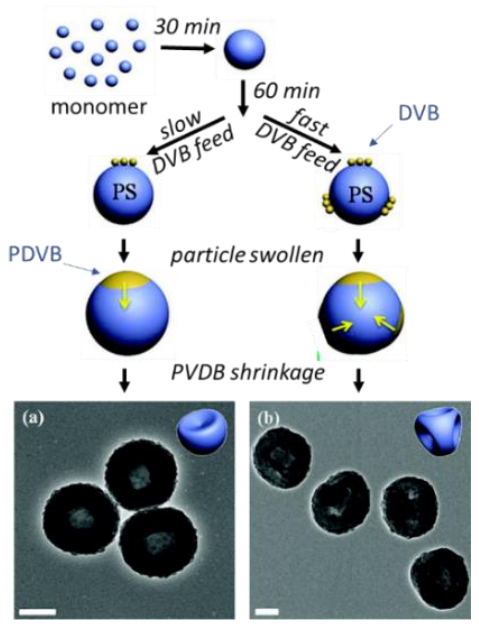

Figure 18 Schematic representation of the preparation of dimpled latex particles and corresponding TEM images showing that the number of cavities depends on the a) slow and b) fast charging mode. Scale bars: $200 \mathrm{~nm}$. Adapted with permission from ref. ${ }^{101}$ Copyright @ 2011, American Chemical Society.

In seeded growth emulsion polymerisation, the protrusions or concavities endowing the patchy character are controlled by phase separation phenomena, which yields to complex entanglements of linear and crosslinked homopolymers and copolymers. Nevertheless, patchy particles at lower scales can be achieved through the reverse way, i.e. the high precision synthesis of block copolymers and their controlled phase separation by solvent evaporation induced assembly within emulsion droplets (3-D confinement) or through selective solvent swelling.

In a first example, the most accessible typical system, i.e. with two blocks usually illustrated by PS and P4VP, is initially dissolved in a good solvent for both blocks, such as chloroform, and the solution was subsequently emulsified in the bad solvent for PS (water) to obtain the patchy particles after evaporating the chloroform. In this way, Deng et al. obtained Janus nanoparticles and showed that their aspect ratio is controlled by the P4VP fraction. ${ }^{102}$ This method is robust without the requisites of narrow molecular weight distribution and a specific range of block fraction of the copolymers. The same authors, ${ }^{103}$ simultaneously to Kim and co-workers, ${ }^{104}$ showed that the strategy could be extended for the synthesis of PS nanoparticles with up to nine P4VP patches by increasing the volume of the emulsion droplet, i.e. the volume of the particle (Fig. 19). For a given particle volume, the morphology can then be precisely tuned by modulating the interfacial interaction at the particle/water interface using a mixture of two different surfactants. ${ }^{104}$ 


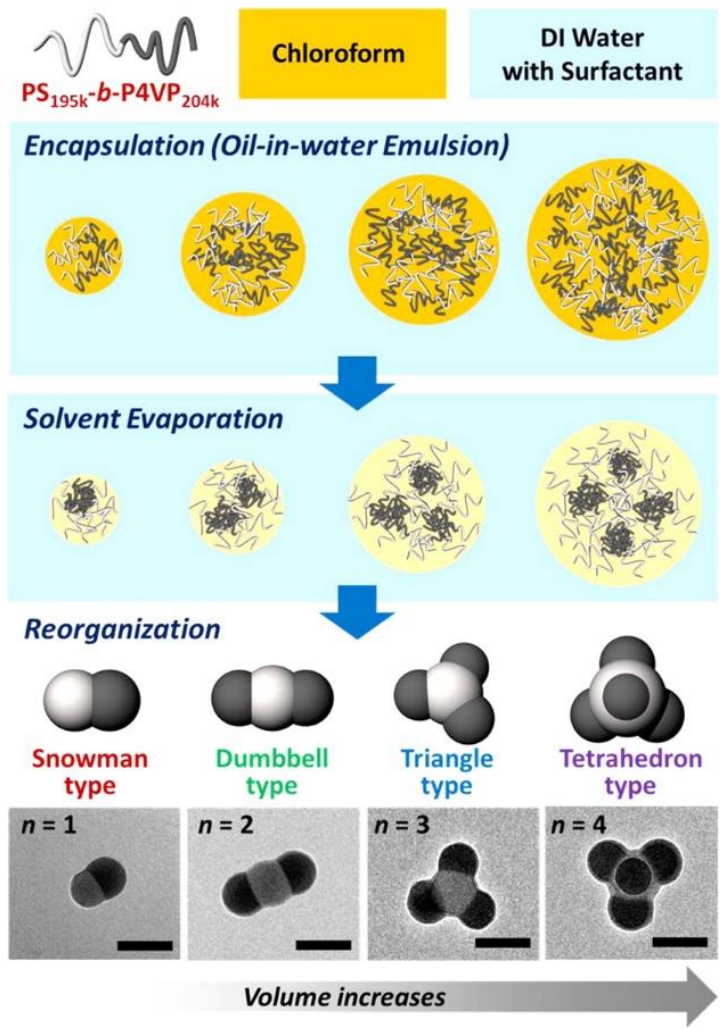

Figure 19 Schematic illustration of the fabrication of soft patchy particles of PS- $b$-P4VP through oil-in-water emulsion evidencing the influence of the volume of the particles on the patch number. Scale bars: $100 \mathrm{~nm}$. Reproduced with permission from ref. ${ }^{104}$ Copyright (c) 2015, American Chemical Society.

The second example concerns well-defined triblock copolymers that do not need confinement conditions to yield to multicomponent patchy nanoparticles. ${ }^{105}$ Gröschel et al. simply played with selective solvent and non-solvent for each block (Fig. 20). The morphology of the as-obtained particles depends on the volume of each unit and the solvent introducing sequence with unprecedented structural control via the intermediate pre-assembly of subunits. This directed selfassembly leads to a stepwise reduction of the degree of conformational freedom and dynamics and avoids undesirable kinetic obstacles during the structure build-up.

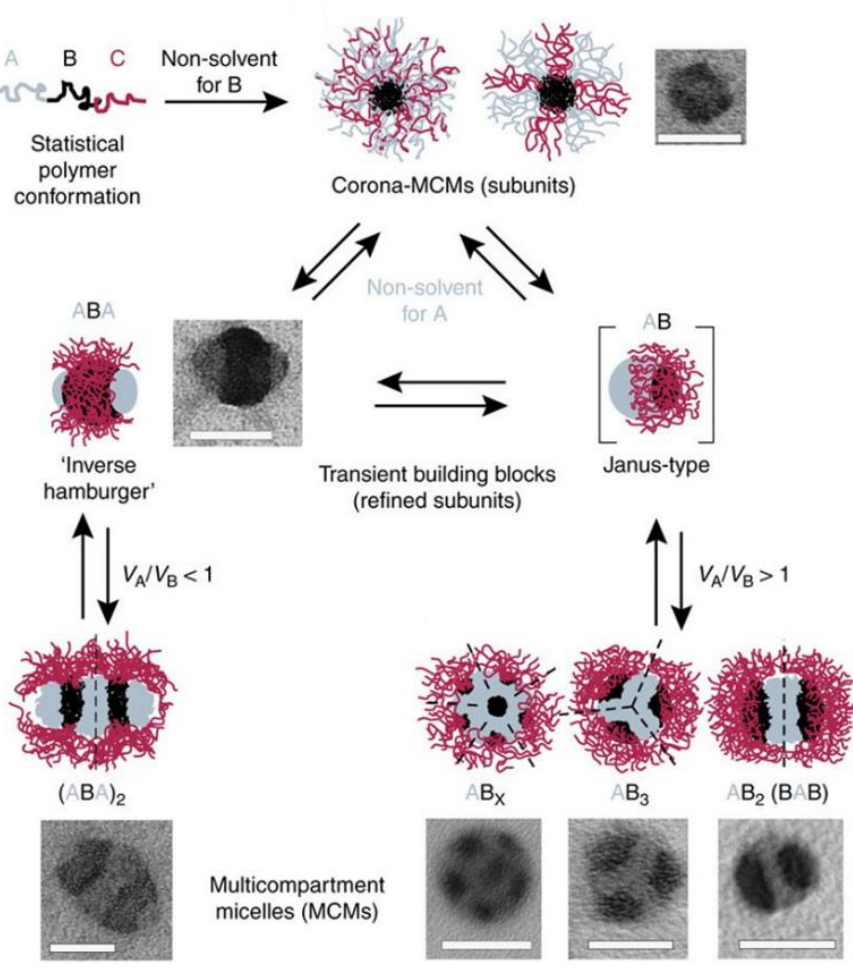

Figure $\mathbf{2 0}$ Schematic illustration of the synthesis pathway of multicompartment micelles from triblock copolymers and corresponding TEM images after $\mathrm{OsO}_{4}$-staining (PS grey, PB black and PMMA not visible due to e-beam degradation). Scale bars: $50 \mathrm{~nm}$. Adapted with permission from ref. ${ }^{105}$ Copyright (c) 2012, Springer Nature.

\section{Assembly of patchy particles into hierarchical structures}

The assembly of patchy particles is expected to yield welldefined structures and especially more open frameworks than isotropic particles do. Nevertheless, the interrelationship between the number of patches and the number of established bonds is not as simple as in the atomic world as demonstrated thanks to the huge amount of simulation studies which have described these last two decades the diversity of the possible structures. ${ }^{106}$ The patch-to-particle size ratio is one of the critical parameters. Roughly, the larger the patch, the higher the number of establishable bonds, especially as the patch number is low. That is why one-patch particles are often multivalent, leading to large clusters or chains, and two-patch particles can be not only chained but also arranged in 2-D structures such as kagome-like lattices.

The aim of this section consists of describing the assemblies that have been experimentally achieved. We focus on the physicochemical and chemical driving forces, e.g. depletion forces, hydrophobic/solvophobic interactions, DNA hybridisation, covalent or supramolecular coupling, meaning that we deliberately omitted the assembly strategies using external fields, such as electric ${ }^{107,108}$ or magnetic ${ }^{109}$ fields. The reader who is particularly interested in assembling particles with magnetic patches can refer to the recent Rossi's review. ${ }^{110}$ For a comprehensive survey of the simulation studies, there exist also several review papers. ${ }^{111-113}$ 


\subsection{Zero-dimension assemblies}

These are clusters obtained through the assembly of one central multipatch particle combined to satellite particles generally to reach new CMs. However, there are also many examples of clusters obtained in an entirely controlled way from the selfassembly of one-patch particles.

\subsubsection{Directed assembly of satellite particles around one multipatch particle}

\section{Depletion forces}

The depletion attraction force was firstly introduced by Asakura and Oosawa through a simple conceptual experiment. ${ }^{114}$ This consists of adding to the liquid dispersion of colloidal particles some depletant agents, which can be smaller particles or macromolecules whose main feature is the lack of interaction with the colloids. When the distance between two colloidal particles is less than the diameter of a depletant, the jointed region is occupied by the pure solvent. So, the osmotic pressure induces the elimination of the pure solvent to the outside region with additives, thereby forming the attraction between the two colloids and forcing their closure. Already exploited for the formation of $\mathrm{CCs},{ }^{115}$ the depletion technique has been recently extended to fabricate clusters from microparticles. Depletion attraction is indeed particularly efficient when the particle shapes are complementary, as evidenced through the lock-and-key principle proposed by Pine and co-workers. ${ }^{83,116,95}$ They used silica microparticles with a well-defined number of concavities (cf. Fig. 15 right) combined to conventional spheres whose diameter fits that of the dimples, in the presence of poly(ethylene oxide) $\left(\bar{M}_{w}=600,000 \mathrm{~g} / \mathrm{mol}\right)$ as depletant. ${ }^{95} \mathrm{AX}$, $A X_{2}, A X_{3}, A X_{4}$, and $A X_{5} C M s$ were stoichiometrically achieved when shifting the monovalent lock to multivalent ones (Fig. 21).

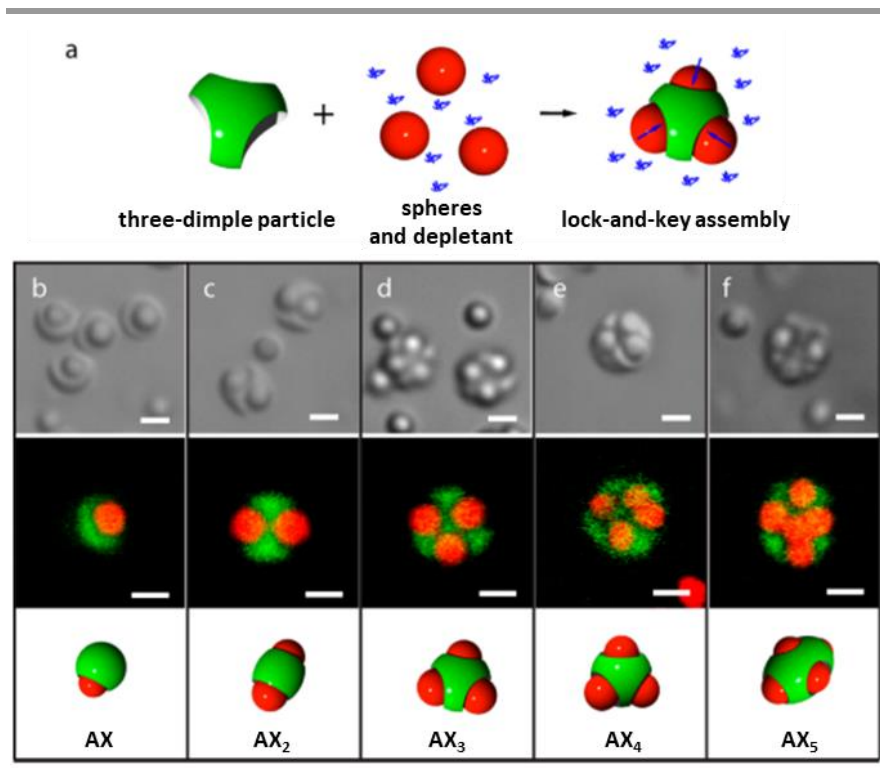

Figure 21 Lock-and-key assemblies. a) Schematic illustration showing a three-patch particle with three assembled spheres using depletion interaction. The depletant (blue coil) causes osmotic pressure (arrows) between adjacent colloids, which is maximised when a sphere assembles into a cavity. b-f) Bright field micrographs (top panel), confocal micrographs (middle panel), and cartoons (bottom panel) showing multivalent lock particles with b) one, c) two, d) three, e) four, and f) five cavities binding to red fluorescent spheres stoichiometrically. Scale bars: $1 \mu \mathrm{m}$. Reproduced with permission from ref..$^{95}$ Copyright (C) 2014, American Chemical Society.

\section{DNA hybridisation}

DNA interaction was utilised for the assembly of particles bearing patches made of complementary strands. A typical example was reported by Pine and co-workers from the patchy particles described in Fig. 15 left. $^{94}$ They obtained new CMs from $A X$ to $A X_{4}$ morphology (Fig. 22). Thanks to a high patch-toparticle size ratio, they also observed cis and trans configurations and $\mathrm{CMs}$, which could mimic ethylene molecules if both carbon atoms are considered embedded in the single oblong colloid. Stepwise self-assembly was demonstrated with similar particles bearing two types of DNA strands, i.e. one type on the patches and another one on the shell, taking advantage of the $10^{\circ} \mathrm{C}$ difference between their melting temperature..$^{96}$

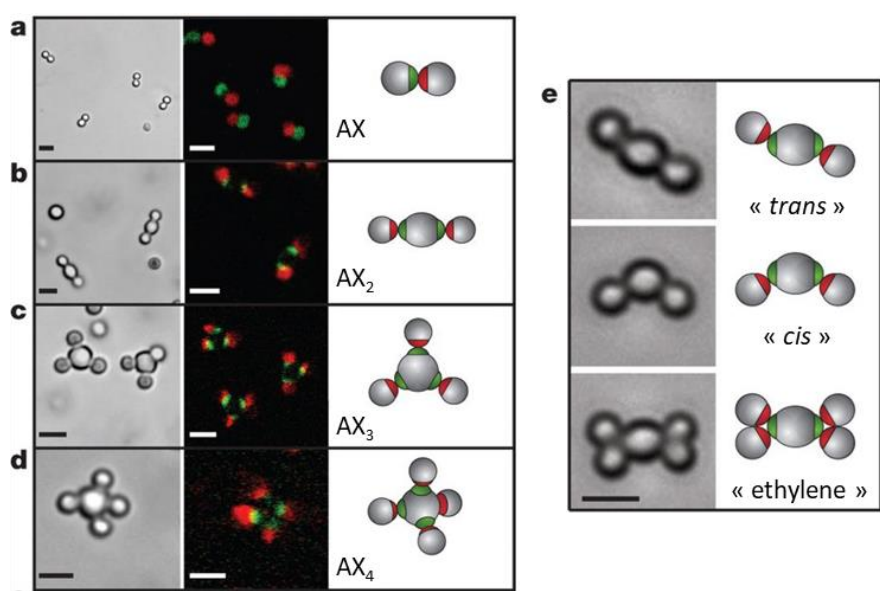

Figure 22 Specific directional bonding between PS microparticles with DNA patches observed with optical microscopes. a-e) Bright-field (left panels), confocal fluorescent (middle panels), and schematic images (right panels) show CMs self-assembled from patchy particles through DNA hybridisation. a) Complementary green and red monovalent particles form dumbbell-shaped $A X$-type molecules. Supra- colloidal molecules $A X_{2}, A X_{3}$ and $A X_{4}$ are formed by mixing red monovalent with green divalent $b$ ), trivalent c) and tetravalent d) particles. e) When particles with larger patches are used, cis-trans-like isomers can form. Introducing more monovalent particles leads to ethylene-like colloidal molecules. Images are bright-field (left panels) and schematic (right panels). Scale bars: $2 \mu \mathrm{m}$. Reproduced with permission from ref. ${ }^{94}$ Copyright (C) 2012, Springer Nature.

\section{Covalent bonding}

Our group successfully obtained silica CMs from the dimpled particles fabricated according to the pathway summarised in Fig. 17. The strategy consists of derivatising the anchored PS macromolecules at the bottom of the dimples to make them first chloromethylated, second aminated. ${ }^{117}$ Then, they are incubated in DMF with an excess of silica NPs whose surface ester groups are activated in order to lock the spheres within the dimples via amide bonds. TEM pictures show that most of the dimples are indeed occupied by the satellite particles in a robust way making separation by centrifugation possible. When the size of the satellite is well adapted, the number of satellites corresponds to the number of patches meaning that in such systems the valence can be controlled simply through the patch number. Different $\mathrm{CMs}$ were obtained from $\mathrm{di}_{-}$, tri- and tetravalent particles and the diversity was also increased when 
two types of silica satellites were simultaneously employed (Fig. 23). ${ }^{118}$ Chiral molecules were observed randomly when using four differently-sized satellites. ${ }^{117}$
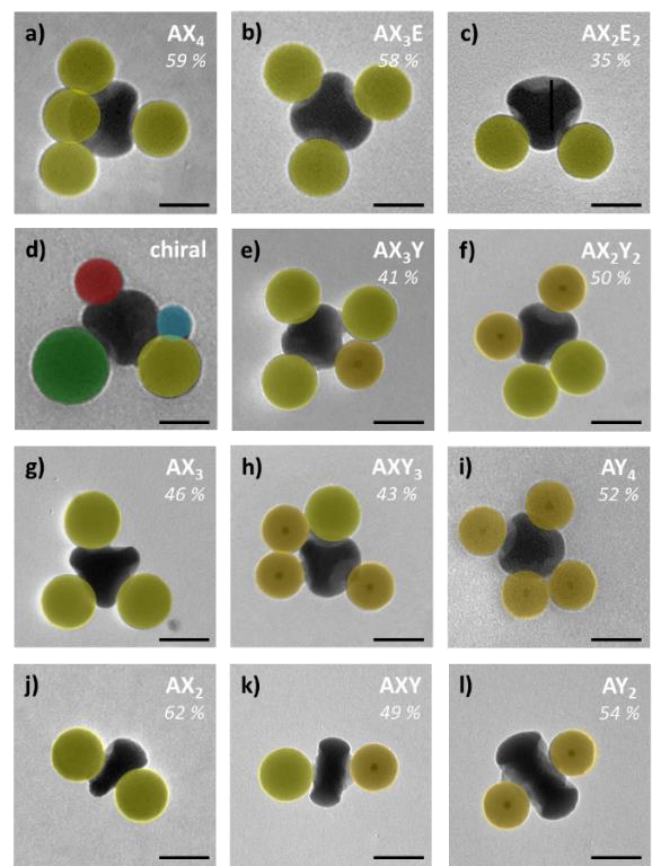

Figure 23 TEM images of silica CMs obtained by directional covalent bonding from patchy particles with four dimples (a-f; $\mathrm{h}-\mathrm{i})$, three dimples (g) and two dimples $(\mathrm{j}-\mathrm{I})$ and satellites of pure silica and different sizes labelled in yellow $(100 \mathrm{~nm})$, red $(80 \mathrm{~nm})$, blue $(60 \mathrm{~nm})$ and green $(130 \mathrm{~nm})$ or $90-\mathrm{nm}$ silica satellites including a central gold dot (labelled in orange). ${ }^{117,118}$ For each image, the terminology refers to the formalism proposed in Fig. 1 and the percentage value indicates the prevalence of the shown morphology knowing that the exact stoichiometry of nanoparticles was employed to obtain it. Scale bars: 100 $\mathrm{nm}$. Adapted with permission from ref. ${ }^{117}$ Copyright (C) 2018, Wiley-VCH Verlag GmbH \& Co. KGaA, Weinheim.

\subsubsection{Controlled aggregation of one-patch particles}

There exist several reported works dedicated to the selfaggregation of one-patch, i.e. Janus-like, particles in the range of $50 \mathrm{~nm}$ to $200 \mu \mathrm{m}$ (Table 1) and the field was recently reviewed. ${ }^{73,74,119}$ The authors generally obtained complex mixtures of fragile clusters prohibiting any purification attempt. This relative instability is due to the too low or reversible attractive forces between the patchy particles. As far as we know, their strengthening by a chemical or physicochemical process was not reported.
Table 1 Some examples of clusters with aggregation number $N$ obtained from one-patch particles.

\begin{tabular}{|c|c|c|c|}
\hline One-patch particles & $\begin{array}{l}\text { Driving force for } \\
\text { assembly }\end{array}$ & $N$ & Ref. \\
\hline $\begin{array}{l}\text { 1- } \mu \mathrm{m} \text { carboxylate-coated PS } \\
\text { spheres then half-coated with gold } \\
\text { treated with (11- } \\
\text { mercaptoundecyl)ammonium } \\
\text { chloride }\end{array}$ & $\begin{array}{l}\text { Electrostatic } \\
\text { attraction }\end{array}$ & $2-12$ & 120 \\
\hline $\begin{array}{l}\text { 1- } \mu \text { m carboxylate-modified PS } \\
\text { spheres half-coated with gold } \\
\text { treated with alkanethiol }\end{array}$ & $\begin{array}{l}\text { Solvophobic } \\
\text { effect helped } \\
\text { with } \\
\text { electrostatic } \\
\text { screening } \\
\end{array}$ & $2-9$ & 121 \\
\hline $\begin{array}{c}\text { 1- } \mu \mathrm{m} \text { sulfate PS spheres half- } \\
\text { coated with gold treated with } \\
\text { alkanethiol }\end{array}$ & $\begin{array}{l}\text { Hydrophobic } \\
\text { attraction and } \\
\text { electrostatic } \\
\text { repulsion }\end{array}$ & $2-7$ & 122 \\
\hline $\begin{array}{l}\text { 4- } \mu \mathrm{m} \text { snowman-like PS particles } \\
\text { with smooth body and rough head }\end{array}$ & $\begin{array}{l}\text { Roughness- } \\
\text { controlled } \\
\text { depletion } \\
\text { attraction }\end{array}$ & $2-12$ & 123 \\
\hline $\begin{array}{l}\text { 3- } \mu \mathrm{m} \text { gold half-coated silica } \\
\text { spheres }\end{array}$ & $\begin{array}{l}\text { At tipping } \\
\text { points of } \\
\text { thermally- } \\
\text { switched } \\
\text { wetting }\end{array}$ & 4- 20 & 124 \\
\hline $\begin{array}{c}\text { 4- } \mu \mathrm{m} \text { iron oxide half-coated latex } \\
\text { spheres }\end{array}$ & $\begin{array}{l}\text { Lipid-induced } \\
\text { capillary } \\
\text { bridging }\end{array}$ & $2-5$ & 125 \\
\hline $\begin{array}{c}\text { 120-nm organosilica sphere with } \\
\text { one } 44-\mathrm{nm} \text { gold head }\end{array}$ & $\begin{array}{l}\text { van der Waals } \\
\text { force and } \\
\text { electrostatic } \\
\text { force }\end{array}$ & $2-3$ & 126 \\
\hline $\begin{array}{l}\text { 50-nm silica sphere with one } 45-\mathrm{nm} \\
\text { gold head coated with alkanethiols }\end{array}$ & $\begin{array}{c}\text { Solvophobic } \\
\text { effect }\end{array}$ & $4-7$ & 127 \\
\hline $\begin{array}{l}\text { 1- } \mu \text { m organosilica sphere with a PS } \\
\text { patch (patch ratio of } 0.13 \text { ) which is } \\
\text { functionalized with DNA having } \\
\text { self-complementary sticky ends }\end{array}$ & $\begin{array}{l}\text { DNA-mediated } \\
\text { attraction } \\
\text { varied by } \\
\text { varying the } \\
\text { temperature }\end{array}$ & $2-4$ & 128 \\
\hline
\end{tabular}

To obtain clusters that are more robust, three studies can be highlighted. Bon and co-workers synthesized asymmetric dumbbells made of a PS microsphere and a poly(nbutylacrylate) lobe and showed their ability to form clusters when dispersed in aqueous media. ${ }^{129}$ The assembly process is induced by the desorption of the poly(vinylpyrrolidone) stabiliser from the particles and the merging of the soft poly $(n-$ butylacrylate) lobes upon contact through collision. More recently, Xu and coll. exploited the drying of Pickering-like water droplets made from 200- $\mu \mathrm{m}$ hydrophilic poly(ethylene glycol) diacrylate sphere with $160-\mu \mathrm{m}$ ethoxylated trimethylolpropane tri-acrylate lobe prepared by a microfluidic technology. ${ }^{130}$ They obtained quite regular submillimetre-sized clusters with aggregation numbers up to eight. Lastly, we recently reported the fabrication of clusters by using silica/PS asymmetric dumbbell-like nanoparticles (Figure 24), ${ }^{131}$ synthesized 
according to a protocol previously reported by Guignard and Lattuada. ${ }^{132}$ The one-patch particles stuck together by their PS lobe after swelling/plasticisation by DMF from ethanol/DMF mixtures at room temperature, leading to clusters with aggregation numbers from 2 to more than 6. The "sticky" regime corresponds to DMF volume fractions of $30-50 \%$ and can be extended to the $10-60 \%$ range, when the assembly is performed under centrifugation.

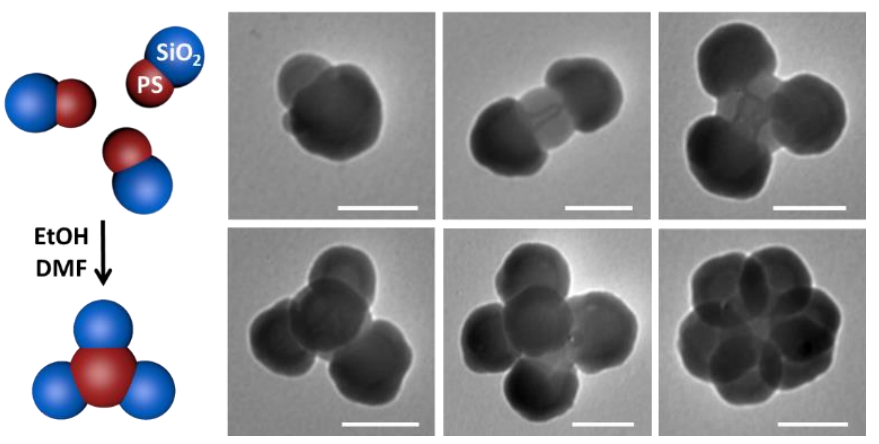

Figure 24 TEM images of CMs made of silica satellites and a PS central core obtained in ethanol/DMF mixture with a DMF fraction of 30 vol.\%. Scale bars: $100 \mathrm{~nm}$. Reproduced with permission from ref. ${ }^{131}$ Copyright (c) 2019, Elsevier Inc.

\subsection{One-dimension assemblies}

Chaining of particles, often described as colloida polymerisation, is a field of intense research that was recently reviewed several times. ${ }^{133-135}$ In the restricted fields of inorganic particles, Pyun and co-workers identified three main strategies based on assembly directed by dipoles, e.g. electrostatic or magnetic, by surface lattice fringes or by surface organic ligands providing electrostatic, covalent or noncovalent linkages, e.g. oligonucleotide. In this last scheme, there exist few studies employing patchy particles for programming a one-direction growth. ${ }^{135}$ We describe some of them below by emphasising those using two-patch particles, i.e. omitting those employing one-patch particles which generally give rise to helices, double-line chains, dimer chains or trimer chains. ${ }^{128,136}$

\subsubsection{From polymer-tethered metal nanorods}

One of the main achievements in this area concern the chaining of metal nanorods. ${ }^{134}$ As previously discussed, gold nanorods can be considered as two-patch NPs because of the different reactivity of their tips compared to the body part. Murphy and co-workers were among the first ones to observe that the density of CTAB ligands is lower at the tips of the gold nanorods making it possible to site-specifically bind biotin disulphide and therefore link the NPs end-to-end by adding streptavidin. ${ }^{137}$ Kumacheva and co-workers enhanced the patchiness of CTABcoated gold nanorods by the functionalization of their tips with thiol-terminated PS ligands, which enabled the formation of linear and cyclic colloidal polymers when dispersed and deposited onto substrates from selective solvent systems. ${ }^{86}$ The driving force to assemble hydrophobic NPs derives from the solvation free energy reduction between the isolated particles and their assembly. ${ }^{138}$ Based on this principle, hydrophobic patches become attractive in binary solvent mixtures with different polarities to decrease the free volume energy. ${ }^{139}$ In this way, the authors succeeded in the self-assembly of the PStethered gold nanorods by using mixtures of one good solvent, e.g. THF or DMF, and one bad solvent for PS, e.g. water or ethanol (Fig 25). ${ }^{86,140}$ Degrees of polymerisation (DP) of up to 50 were observed by TEM for linear chains of nanorods endfunctionalized with PS ligands $\left(\bar{M}_{n}=30,000 \mathrm{~g} / \mathrm{mol}\right)$ and cast from DMF/water dispersions. ${ }^{141}$ Copolymer-like assemblies were also achieved when mixing gold and palladium nanorods. ${ }^{142}$ The colloidal polymerisation was shown to follow step-growth polymerisation models, and this was the first quantification attempt using classical polymerisation approaches. ${ }^{140}$ They also developed the concept of colloidal chain stoppers for controlling DP by using snowman-like particles made of an iron oxide body and gold head subjected to a two-step ligand exchange to cap them with dopamineterminated polyethylene glycol and thiol-terminated PS macromolecules, respectively. ${ }^{143}$ Lastly, the authors reported that in the presence of salts, the major driving force for selfassembly is a decrease in solubility of the PS ligands rather than a screening of the electrostatic repulsion. ${ }^{144}$ Photo-crosslinking of poly(styrene-co-isoprene) end-functionalized gold nanorods was also demonstrated as a useful means to covalently fix the bond angles in the chains. ${ }^{145}$ 
a)

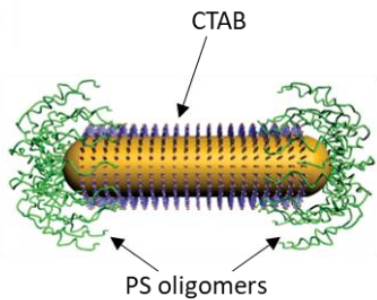

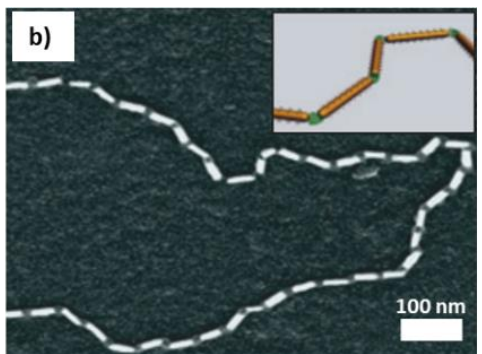
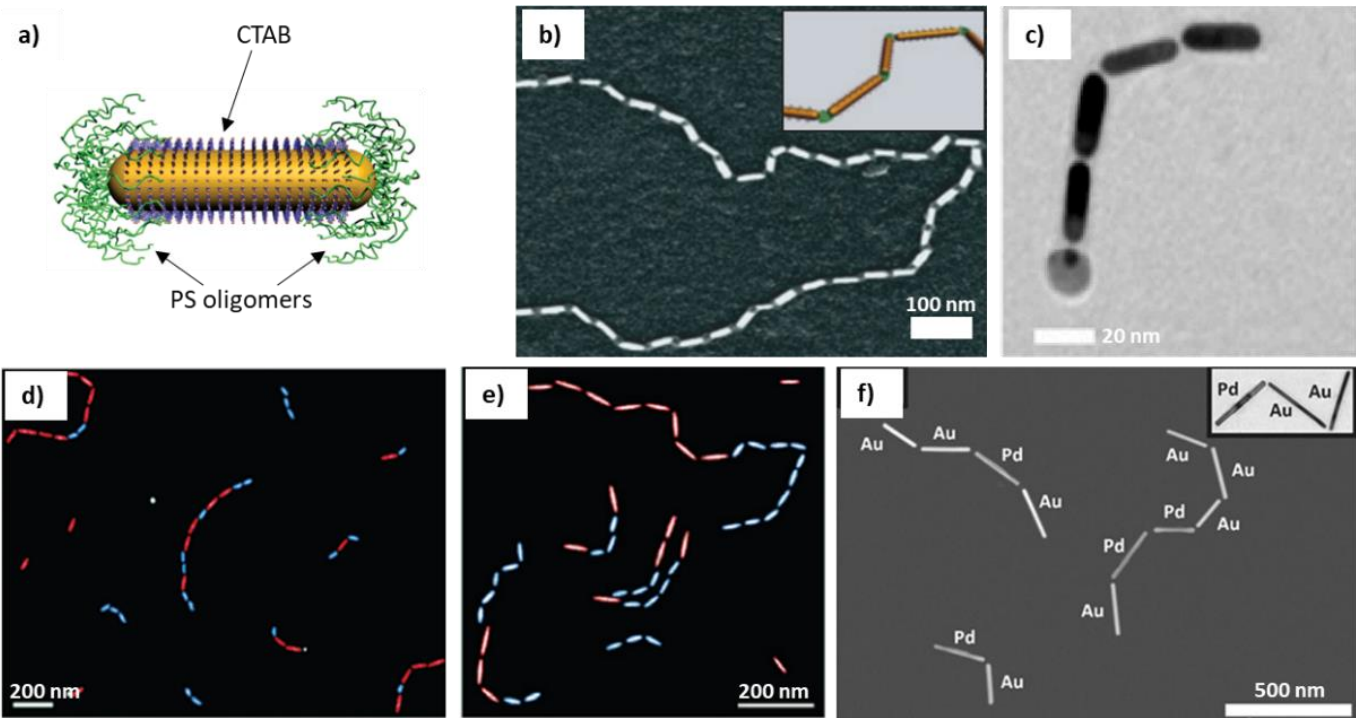

Figure $\mathbf{2 5}$ a) schematic representation of gold nanorods where CTAB ligands at the tips have been replaced by thiol-terminated PS chains; TEM images of b) a long chain after endto-end assembly, c) a chain terminated by a chain stopper, d) random copolymers and e) block-copolymers from nanorods of two different lengths, and f) random copolymers from gold and palladium nanorods. Adapted with permission from ref. ${ }^{86}$ Copyright $@ 2007$, Springer Nature. Adapted with permission from ref. ${ }^{143}$ Adapted with permission from ref. ${ }^{142}$ Copyright @ 2014, WILEY - VCH Verlag GmbH \& Co. KGaA, Weinheim.

Alternatively, gold nanorods functionalised at both ends with thiol-terminated PNIPAM $\left(\mathrm{M}_{\mathrm{n}}=13,000 \mathrm{~g} / \mathrm{mol}\right)$ ligands were also demonstrated to undergo (photo)thermally triggered selfassembly to form linear and branched colloidal polymers. ${ }^{146}$ The NPs were heated above the lower critical solution temperature (LCST) of PNIPAM to induce colloidal polymer formation with an average DP of 11. Similar chaining was observed when the LCST was reached upon illumination at a wavelength near the longitudinal plasmon band of the nanorods.

More recently, Weizmann and co-workers implemented a DNA-based strategy to design gold nanorods with one or two sticky ends through partial polymer blocking followed by modification of the polymer-free surface region with singlestrand DNA (Fig. 26 left). ${ }^{147}$ They achieved nanorods chaining by self-assembly dictated by the hybridization of DNA into preconceived linear structures (Fig. 26 right). The authors extended the assembly of metallic nanoparticles to other shapes (nanospheres, nanocubes, triangular nanoprisms) and chemical natures (palladium). A total of 24 complex nanoassemblies were fabricated, evidencing the versatility of the proposed strategy.

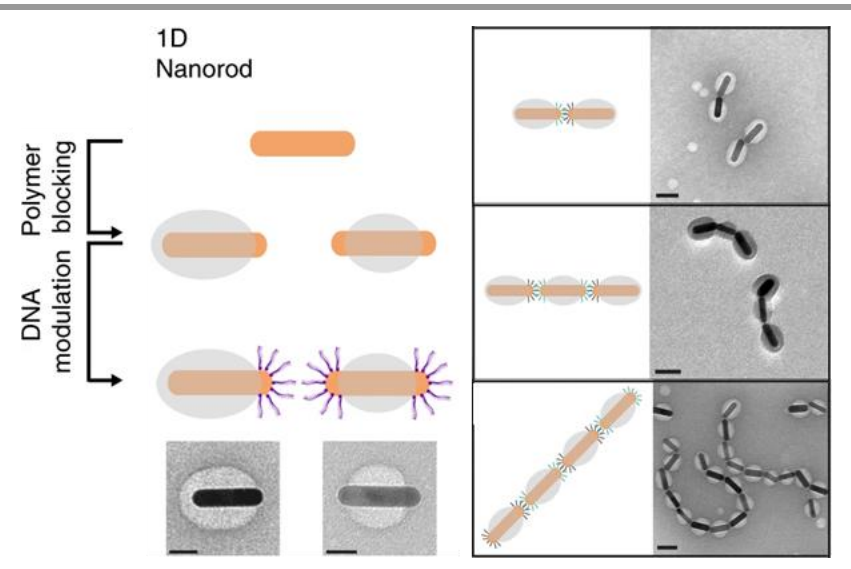

Figure 26 Left: scheme showing the general strategy to synthesize gold nanorods with one or two sticky ends and TEM images of the so-obtained nanoparticles. Scale bars: 20 $\mathrm{nm}$. Right: schematic representation and TEM images the self-assembled structures from the combination of different nanorods. Scale bars: $50 \mathrm{~nm}$. Adapted with permission from ref. ${ }^{147}$ Copyright (c) 2018, Springer Nature.

\subsubsection{From particles with two concave or convex patches}

According to the lock-and-key concept, Sacanna, Pine and coworkers subjected organosilica microspheres bearing one dimple to depletion conditions and observed their stacking when the diameter of the dimples fits that of the other hemisphere. ${ }^{116}$ The absence of irreversible chemical bonds between the building blocks allows these ball-in-socket joints to move freely and confers high flexibility to the colloidal macromolecules. Similar results were obtained by Tigges and Walther by using cone-shaped microparticles fabricated by direct 3-D laser writing which consists in crosslinking a resin through two-photon lithography. ${ }^{148}$

Pine and coll. also obtained short chains from two-patch organosilica microparticles and DNA hybridisation. ${ }^{94,96}$ With the same microparticles, the idea was extended by Weck and coworkers to liquid-assisted capillary bridging ${ }^{97}$ and reversible supramolecular coupling by metal coordination, ${ }^{149}$ host-guest pairing cucurbit[7]uril and viologen motifs not only lightmediated, ${ }^{150}$ but also redox-mediated. ${ }^{151}$ Nevertheless, DP generally remained generally below 12 and branches due to the simultaneous interaction of three patches were frequently observed.

\subsubsection{From multicomponent macromolecular micelles}

The ABC triblock NPs prepared by Gröschel et al. (cf. Fig. 20) were successfully employed to obtain long and highly regular chains thanks to selective solvents. ${ }^{152}$ Briefly they showed that SDM tri-block copolymers made of polystyrene-block-poly(3butenyl(dodecyl)sulphane)-block-poly(methyl methacrylate) 
for which the volume ratio of $S$ and $D$ segments, $V_{S} / V_{D}$, is $<1$, are changed first into core-corona colloids where the $S$ blocks create two opposite patches in the presence of a non-solvent of the central blocks, and second into linear chains once the solvent quality has been reduced to a critical threshold for the $S$ patches (Fig. 27a). The authors prepared hybrid materials by selectively encapsulating $10-\mathrm{nm}$ magnetite NPs within the D segments of SDMS at the first stage (Fig. 27b) and extended this approach to design multiblock co-assemblies by combining two divalent units, i.e. $\mathrm{SD}^{\mathrm{M} S}$ and $\mathrm{SB}^{\mathrm{M} S}$ where $\mathrm{B}$ is a polybutadiene block (Fig. 27c). They also got many other complex morphologies. ${ }^{152}$

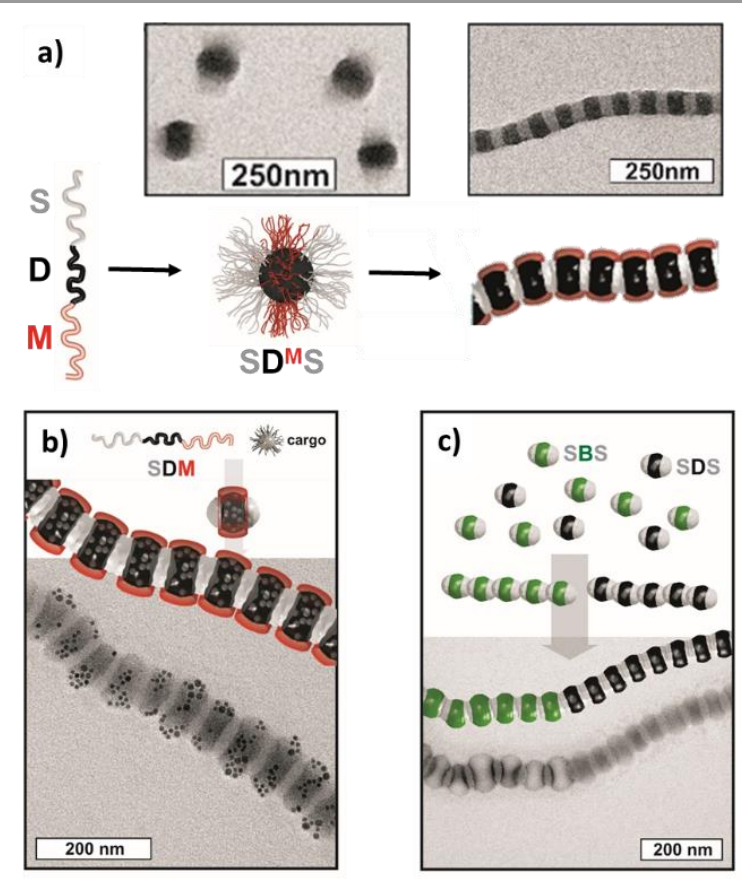

Figure $\mathbf{2 7}$ Hierarchical assembly of triblock copolymers in selective solvents: a) schematic representation of the two-stage process and corresponding TEM images, b) hybrid co assemblies with 10-nm magnetite NPs selectively loaded within the D segment, and c) linear multiblock co-assembly composed of $\left[\mathrm{SD}^{\mathrm{M} S}\right]_{\mathrm{m}} /\left[\mathrm{SB}^{\mathrm{M} S}\right]_{\mathrm{n}}$ sequences ( $\mathrm{M}$ corona omitted for clarity). Adapted with permission from ref. ${ }^{152}$ Copyright (c) 2013, Springer Nature.

In the same vein, Sohn and coll. reported recently the preparation and polymerisation of two-patch micelles obtained from PS-P4VP macromolecules after crosslinking of the P4VP core with 1,4-dibromobutane (Fig. 28a) and dispersion in DMF a better solvent for P4VP than for PS - rearranging the PS corona into two separate patches (Fig. 28b). ${ }^{153}$ Then, the polymerisation is triggered by adding water (Fig. 28c). The possibility to reach block or random copolymers was demonstrated using two differently-sized micelles, i.e. with different block lengths (Fig. 28d-e) or micelles labelled with two types of quantum dots (Fig. 28f-i). ${ }^{154}$ Lastly, branching and crosslinking were introduced in a controlled way by employing three-patch micelles generated when the PS-to-P4VP volume ratio was increased. ${ }^{155}$

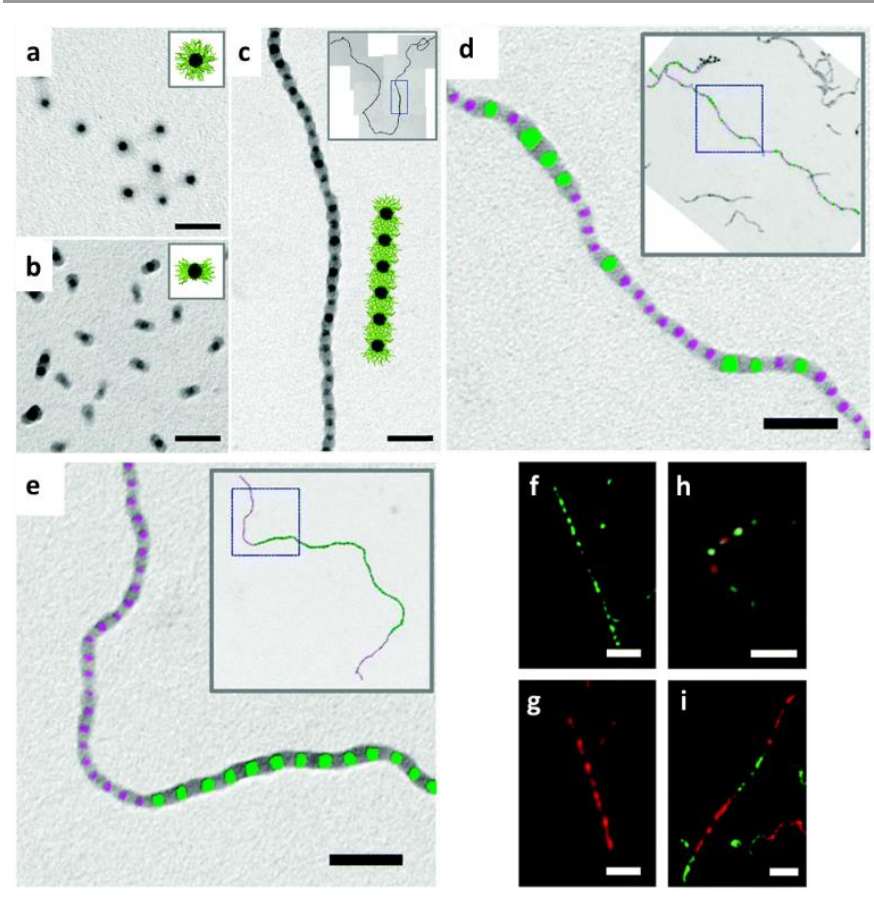

Figure 28 Polymerisation of patchy micelles: TEM images of a) spherical micelles of PS(51)-P4VP(18); b) two-patch micelles obtained after DMF addition, c) colloidal macromolecules obtained after water addition, d) random and e) block copolymer chains obtained from PS(51)-P4VP(18) and PS(25)-P4VP(7) micelles (false colours for clarity), ${ }^{153}$ and structured illumination microscopy (SIM) images of homopolymers from micelles bearing $\mathrm{f}$ ) green and $\mathrm{g}$ ) red quantum dots, $\mathrm{h}$ ) random copolymers and i) block copolymers from the same fluorescent micelles. ${ }^{154}$ Scale bars for TEM and SIM images are $100 \mathrm{~nm}$ and $1 \mu \mathrm{m}$, respectively. Reproduced from ref. ${ }^{153,154}$ with permission from the Royal Society of Chemistry.

\subsection{Two-dimension assemblies}

The well-known 2-D kagome structure reported by Granick and co-workers eight years ago was obtained from microparticles bearing only two patches which were sufficiently large to interact each with two others, ${ }^{156}$ as demonstrated in silico by Romano et Sciortino (Fig. 29). ${ }^{157,158}$ They showed that selfassembly occurs when the strength of the interaction between patches (depicted in red) is large enough to overcome the random forces arising from the thermal jiggling of the particles. In this case, they crystallise into a kagome lattice or a closedpacked triangular lattice at higher density. Experimentally, the two-patch microparticles, improperly called triblock Janus particles, were made up of an electrically charged band inbetween two hydrophobic caps of the proper size and fabricated by the double metal deposition and intermediary particle stamping techniques. After sedimentation, a salt solution allowed to shield the repulsion force and slowly induced the self-assembly. 

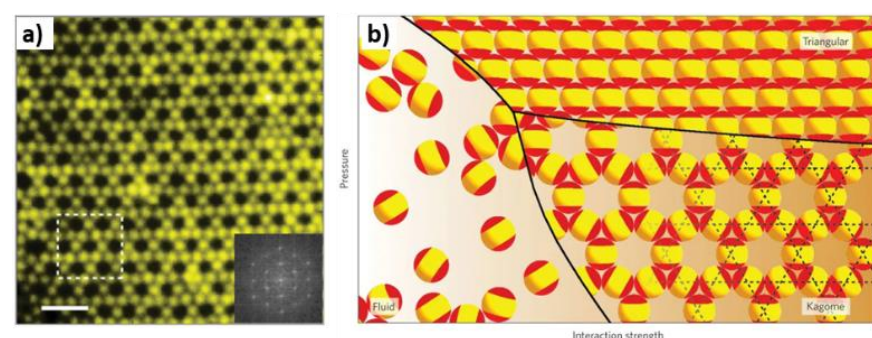

Figure 29 2-D kagome structure obtained by two-patch microparticles self-assembly: a) fluorescence image of the obtained structure ${ }^{156}$ and $b$ ) snapshot of the forming assembly obtained by simulation. ${ }^{158}$ Scale bar: $4 \mu \mathrm{m}$. Reproduced with permission from ref. ${ }^{156,158}$ Copyright $@ 2011$, Springer Nature.

As far as we know, there is no concrete example of colloidal monolayer made of three-patch particles and only Monte-Carlo simulations were reported.158-163 Doppelbauer et al. investigated in a two-dimensional system the self-assembly scenario of particles with three attractive patches. ${ }^{159}$ They identified two minimum energy configurations: at low pressure, honeycomb structure where all patches are saturated via bonds, and at high pressure, a 2-D hexagonal structure where the cores of the particles are close-packed. The number of bonds that the particles can form via the patches plays a minor role in the energetic considerations (Fig. 30a). Sciortino and coworkers identified at low $\mathrm{T}$ three different fully bonded structures: a collection of independent honeycomb-like planes, a crystal consisting of inter-penetrating honeycomb-like planes and a fully bonded $f c c$ structure if the patches are wide enough (Fig. 30b). ${ }^{160}$

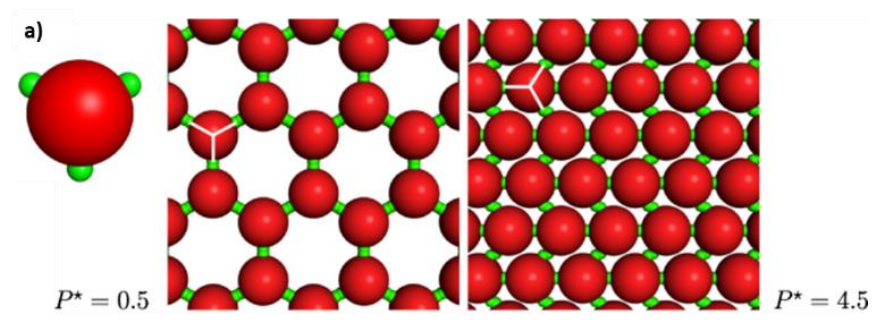

b)

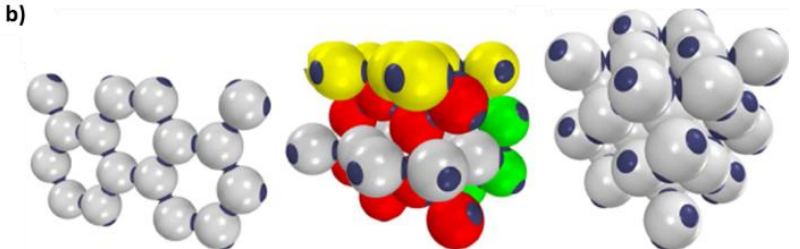

Figure 30 Simulation of assemblies from three-patch particles: a) two minimum energy configurations depending on pressure when constrained in a two-dimensional system, ${ }^{159}$ and b) schematic representation of the crystals examined in a three-dimensional system, i.e. independent honeycomb-like planes, a crystal consisting of inter-penetrating honeycomb-like planes and a fully bonded fcc structure. For better visualisation of the structure, particles in the $2^{\text {nd }}$ phase are coloured according to the plane they belong to. ${ }^{160}$ Adapted with permission from ref. ${ }^{159}$ and reproduced with permission from ref. ${ }^{160}$ C IOP Publishing Inc.

Noya et al. confirmed the existence of the honeycomb lattice at moderately low temperature and pressure, whereas the system would form a close-packed triangular lattice at high temperature and pressure. ${ }^{162}$ When the half-opening angle of the patches is lower than $10^{\circ}$, the fluid transforms upon increasing the pressure into a rather exotic phase, i.e. honeycomb lattice whose voids are filled continuously with additional particles that remain, on average, unbound and capable of free rotation (Fig. 31 top). At moderately low temperature, the fluid transforms into a nearly empty honeycomb lattice, whereas at high temperature it transforms directly into the almost filled lattice. Interestingly, for particles with big patches (with a half opening angle of $20^{\circ}$ ), the honeycomb-like and triangular lattices are separated by a liquid phase that remains stable down to fairly low temperatures (Fig. 31 bottom). Less surprisingly, only particles with big patches exhibit an equilibrium gas-liquid separation. 


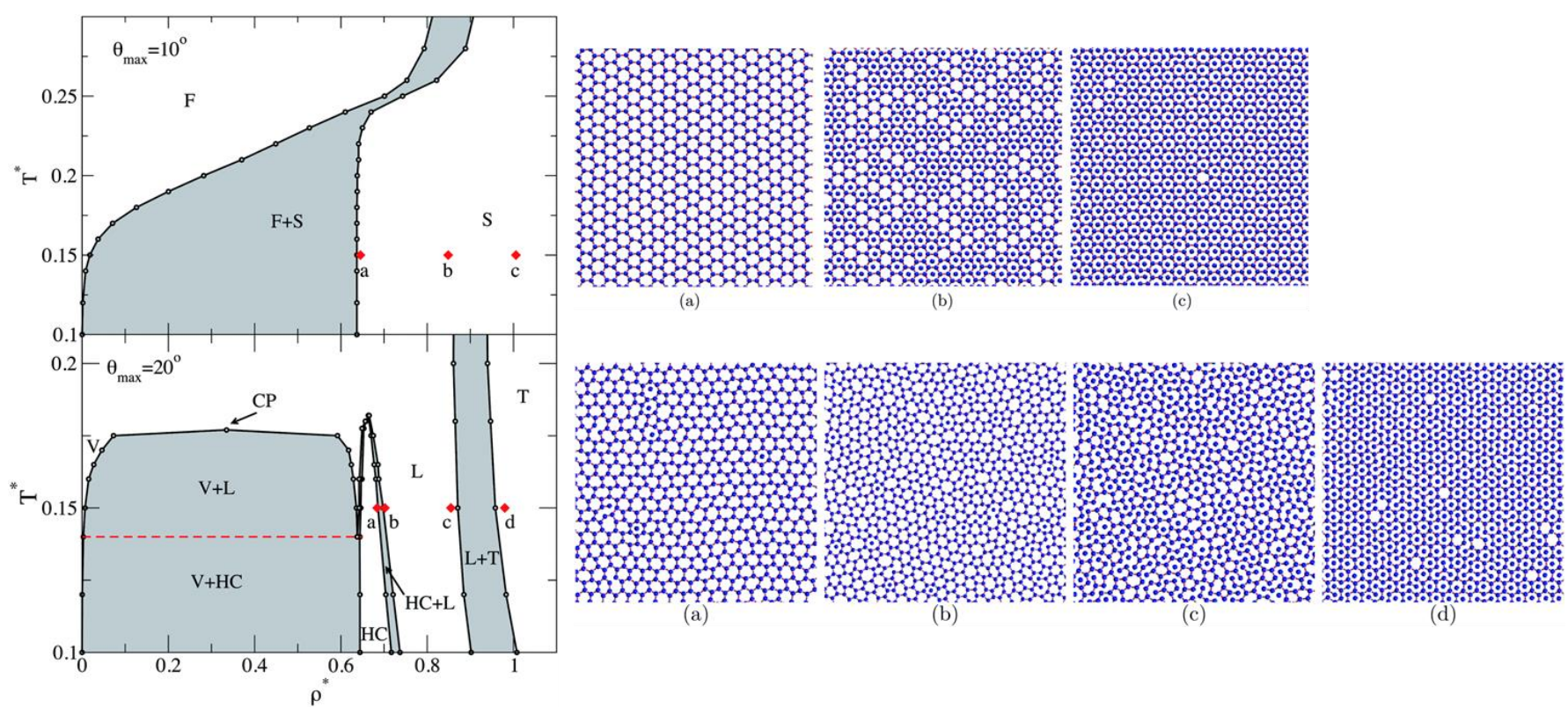

Figure 31 Simulation of assemblies from three-patch particles: T- $\rho$ phase diagram of three-patch particles with small patches (top panel) and big patches (bottom panel). Regions of coexistence of two phases are shown in grey. For particles with small patches, there are only two stable phases, namely, the fluid (F) and the solid (S). For particles with big patches, besides the vapour ( $\mathrm{V}$ ) and the liquid (L), there are two stable solid phases, namely, the honeycomb $(\mathrm{HC})$ and the triangular ( $\mathrm{T}$ ) solids. The horizontal dotted red line marks the location of a triple point at which the vapour, the liquid and honeycomb solids coexist. The critical point (CP) is also labelled. Red diamonds indicate the state points corresponding to the configurations depicted in the right. Adapted from ref. ${ }^{162}$ with permission from the Royal Society of Chemistry.

More recently, Sun and co-workers developed for dynamics simulations a model specific to soft patchy particles, ${ }^{161}$ and Torquato and co-workers generalized inverse techniques to design experimentally realizable spherical colloidal particles with optimized "patchy" anisotropic interactions for a wide class of targeted low-coordinated two-dimensional crystal structures, e.g. square, honeycomb, kagome, and parallelogrammic crystals, that are defect-free. ${ }^{163}$

From an experimental viewpoint, the synthesis of polymertethered gold triangular nanoprisms by ligand island formation specifically at their tips was recently reported by Chen and coll. ${ }^{164}$ Nevertheless their ability to self-assemble into extended 2-D structures has not yet been demonstrated.

\subsection{Three-dimension assemblies}

Patchy particles were originally invented by Glotzer and coworkers in 2005 to solve the problem of the in silico crystallisation of diamond structures. ${ }^{165}$ They indeed envisioned particles with four sticky patches in a tetrahedral arrangement and showed by Monte Carlo simulation that the diamond structure from the disordered state could be obtained (Fig. 32). Crystallisation is greatly favoured by "seeding" the system with small diamond crystallites or by introducing a rotation interaction to mimic a carbon-carbon antibonding interaction. From this time, many complementary simulation papers were published from several groups because photonic crystals based on a diamond structure are expected to present a full photonic bandgap opening avenues to numerous applications. These studies showed that for such four-patch spheres the gas-liquid phase separation is metastable. Indeed, the phase diagram of tetrahedral patchy particles was found to be very rich, with several re-entrant coexistence lines and critical influence of the patch-to-particle size ratio. ${ }^{166}$ It appears that the self-assembly of tetrahedral patchy particles into a diamond-like crystal might not be so straightforward. Lastly, Glotzer and co-workers introduced the notion of entropically patchy particles and showed how specific self-assembled structures could be specifically targeted by engineering directional entropic forces through the systematic alteration of particle shape. ${ }^{167}$ They demonstrated that tetrahedrally faceted spheres, i.e. with four concave patches, crystallise to a diamond lattice when the amount of faceting exceeds a certain value. Patra and Tkachenko recently reported that the self-assembly of a highquality cubic diamond lattice can be achieved by using eight distinct types of chiral particles bearing four different tetrahedrally arranged patches. ${ }^{168}$ They also showed that a slight modification of the pair interactions between patches gives rise to the formation of alternative polymorph, i.e. hexagonal diamond.

However, as far as we know, there is no concrete realisation of diamond-like CCs using four-patch particles. Some artifices were used to reach the goal without patchy particles, such as the guidance of tetrahedral DNA origami, in which gold NPs were entrapped. ${ }^{169}$ 

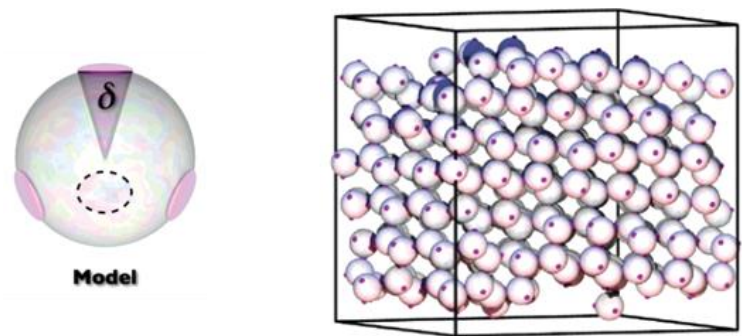

Figure 32 The original model of patchy particle and its assembly into the diamond-like structure through simulation. Adapted with permission from ref. ${ }^{165}$ Copyright $\odot$ 2005, American Chemical Society.

\section{Conclusions}

The concepts of colloidal molecules and patchy colloids are now intimately linked, since some may be the precursors of others and vice versa. However, the assembly capabability of the patchy particles are more promising and should make it possible to obtain functional materials in the shorter term.

Chemists have redoubled their efforts to create ever more complex particles, even if the yield of the recipes and the purity of the products are not systematically discussed. DNA hybridisation is now a mature technology for assembling particles together. It is now also aimed at large particles and the most remarkable achievements concern the DNA origami belt ${ }^{41}$ and surface mobile DNA linkers. ${ }^{42}$

The assembly of patchy particles has already made it possible to imitate simple molecules most often made of a single central atom, or chains whose monomer unit consists of a single atom, such as carbyne. Imitating slightly more complex macromolecules such as a polyacetylene or polyethylene chain remains an obstacle to overcome and the solution will undoubtedly pass in the decoupling of the assembly of the chain and that of the substituent atoms, as demonstrated once by Pine, Weck and coworkers. ${ }^{96}$

The higher the dimension of the expected assembly, the more rare are the examples because the higher demanding the purity of the patchy particles in terms of the geometry and size and the higher the degree of reversibility needed to self-heal the stacking errors. This means that on one side the synthesis of elementary bricks and/or purification methods will have to be highly efficient, and on the other side the patch-to-patch attractive forces will have to be selective and quantitative while being partially reversible. This disqualifies the vast majority of current covalent coupling strategies and leaves the DNA hybridisation a good step ahead. Fortunately, the chemists are strongly motivated to progress in these directions not only because the results of the computer simulations are sufficiently exciting in terms of the variety of attainable structures, but also because of the good match between simulations and concrete experiments for instance for the kagome lattices. ${ }^{156,158}$

One of the main trends is to transpose validated concepts with microparticles at a lower scale, especially particles of a few hundred nanometers, in order to see some optical properties materialize in the visible domain. To the synthesis difficulties are then added those related to (i) the assembly forces because the depletion attraction is probably less efficient at these scales and (ii) the assembly monitoring with the obligation to drop optical microscopy in favor of electron microscopy which is significantly more complex to implement especially with liquid cells.

Lastly, the conversion of these concepts into innovative materials will pass either through chemical consolidation steps if the assembly results from entropic mechanisms such as depletion forces, or by the development of assembly paths by covalent bonds as long as they are partially reversible.

\section{Conflicts of interest}

There are no conflicts to declare.

\section{Acknowledgements}

This work was supported by the Agence Nationale de la Recherche (ENLARgER project, ANR-15-CE09-0010, HotChpot project, ANR-17-CE09-0048 and POESY project, ANR-18-CE090019), the LabEx AMADEus (ANR-10-LABX-42) and IdEx Bordeaux (ANR-10-IDEX-03-02), that is, the Investissements d'Avenir programme of the French government managed by the Agence Nationale de la Recherche. Weiya Li was supported by a grant from the China Scholarship Council.

\section{References}

1 T. Palberg, J. Phys. Condens. Matter, 2014, 26, 333101.

2 S. Sacanna and D. J. Pine, Curr. Opin. Colloid Interface Sci., 2011, 16, 96.

3 V. R. Dugyala, S. V. Daware and M. G. Basavaraj, Soft Matter, 2013, 9, 6711.

4 P. F. Damasceno, M. Engel and S. C. Glotzer, Science, 2012, 337, 453.

5 S. Sacanna, D. J. Pine and G.-R. Yi, Soft Matter, 2013, 9, 8096.

6 D. Morphew and D. Chakrabarti, Curr. Opin. Colloid Interface Sci., 2017, 30, 70.

$7 \quad$ W. Xu, Z. Li and Y. Yin, Small, 2018, 14, 1801083.

8 A. van Blaaderen, Science, 2003, 301, 470.

9 A. van Blaaderen, Nature, 2006, 439, 545.

10 A. B. Pawar and I. Kretzschmar, Macromol. Rapid Commun., 2010, 31, 150.

11 H. Rezvantalab and S. Shojaei-Zadeh, Phys. Chem. Chem. Phys., 2014, 16, 8283.

12 S. Ravaine and E. Duguet, Curr. Opin. Colloid Interface Sci., 2017, 30, 45.

13 V. N. Manoharan, M. T. Elsesser and D. J. Pine, Science, 2003, 301, 483.

14 F. Li, D. P. Josephson and A. Stein, Angew. Chemie Int. Ed., 2011, 50, 360.

15 E. Duguet, A. Désert, A. Perro and S. Ravaine, Chem. Soc. Rev., 2011, 40, 941.

16 C. S. Plüisch and A. Wittemann, Macromol. Rapid Commun., 2013, 34, 1798.

17 E. Elacqua, X. Zheng, C. Shillingford, M. Liu and M. Weck, Acc. 
Chem. Res., 2017, 50, 2756.

18 B. Yu, H. Cong, Q. Peng, C. Gu, Q. Tang, X. Xu, C. Tian and F. Zhai, Adv. Colloid Interface Sci., 2018, 256, 126.

19 Y.-S. Cho, G.-R. Yi, S.-H. Kim, D. J. Pine and S.-M. Yang, Chem. Mater., 2005, 17, 5006.

20 V. N. Manoharan, Solid State Commun., 2006, 139, 557.

21 E. Lauga and M. P. Brenner, Phys. Rev. Lett., 2004, 93, 238301.

22 D. Zerrouki, B. Rotenberg, S. Abramson, J. Baudry, C.

Goubault, F. Leal-Calderon, D. J. Pine and J. Bibette, Langmuir, 2006, 22, 57.

23 I. Schwarz, A. Fortini, C. S. Wagner, A. Wittemann and M. Schmidt, J. Chem. Phys., 2011, 135, 244501.

24 D. J. Kraft, W. S. Vlug, C. M. van Kats, A. van Blaaderen, A. Imhof and W. K. Kegel, J. Am. Chem. Soc., 2009, 131, 1182.

25 V. Meester, R. W. Verweij, C. van der Wel and D. J. Kraft, ACS Nano, 2016, 10, 4322.

26 Q. Yuan, J. Gu, Y. Zhao, L. Yao, Y. Guan and Y. Zhang, ACS Macro Lett., 2016, 5, 565.

27 L. Yao, Q. Li, Y. Guan, X. X. Zhu and Y. Zhang, ACS Macro Lett., 2018, 7, 80.

28 B. Shen, J. Ricouvier, F. Malloggi and P. Tabeling, Adv. Sci., 2015, 3, 1.

29 R. W. Perry and V. N. Manoharan, Soft Matter, 2016, 12, 2868.

30 A. F. Demirörs, P. P. Pillai, B. Kowalczyk and B. A. Grzybowski, Nature, 2013, 503, 99.

31 A. P. Alivisatos, K. P. Johnsson, X. Peng, T. E. Wilson, C. J. Loweth, M. P. J. Bruchez and P. G. Schultz, Nature, 1996, 382, 609.

32 F. A. Aldaye and H. F. Sleiman, J. Am. Chem. Soc., 2007, 129, 4130.

33 A. J. Mastroianni, S. A. Claridge and A. P. Alivisatos, J. Am. Chem. Soc., 2009, 131, 8455.

34 X. Xu, N. L. Rosi, Y. Wang, F. Huo and C. A. Mirkin, J. Am. Chem. Soc., 2006, 128, 9286.

35 N. B. Schade, M. C. Holmes-Cerfon, E. R. Chen, D. Aronzon, J. W. Collins, J. A. Fan, F. Capasso and V. N. Manoharan, Phys. Rev. Lett., 2013, 110, 148303.

36 J. T. McGinley, I. Jenkins, T. Sinno and J. C. Crocker, Soft Matter, 2013, 9, 9119.

37 J. T. McGinley, Y. Wang, I. C. Jenkins, T. Sinno and J. C. Crocker, ACS Nano, 2015, 9, 10817.

38 Y. Tian, T. Wang, W. Liu, H. L. Xin, H. Li, Y. Ke, W. M. Shih and O. Gang, Nat. Nanotechnol., 2015, 10, 637.

39 W. Liu, J. Halverson, Y. Tian, A. V. Tkachenko and O. Gang, Nat. Chem., 2016, 8, 867.

40 Y. Li, Z. Liu, G. Yu, W. Jiang and C. Mao, J. Am. Chem. Soc., 2015, 137, 4320.

41 M. Y. Ben Zion, X. He, C. C. Maass, R. Sha, N. C. Seeman and P. M. Chaikin, Science, 2017, 358, 633.

42 I. Chakraborty, V. Meester, C. Van Der Wel and D. J. Kraft, Nanoscale, 2017, 9, 7814.

43 F. Ma, D. T. Wu and N. Wu, J. Am. Chem. Soc., 2013, 135, 7839.

44 F. Ma, S. Wang, D. T. Wu and N. Wu, Proc. Natl. Acad. Sci., 2015, 112, 6307.

45 H. Löwen, Europhys. Lett., 2018, 121, 58001
46 J. M. Asua, J. Polym. Sci. Part A Polym. Chem., 2004, 42, 1025.

47 J. M. Stubbs and D. C. Sundberg, Prog. Org. Coatings, 2008, 61, 156.

48 J. Kim, R. J. Larsen and D. A. Weitz, J. Am. Chem. Soc., 2006, 128, 14374.

49 J.-W. Kim, R. J. Larsen and D. A. Weitz, Adv. Mater., 2007, 19, 2005.

50 J.-G. Park, J. D. Forster and E. R. Dufresne, Langmuir, 2009, 25, 8903.

51 B. Peng, A. van Blaaderen and A. Imhof, ACS Appl. Mater. Interfaces, 2013, 5, 4277.

52 M. Yang, G. Wang and H. Ma, Chem. Commun., 2011, 47, 911.

53 A. Désert, I. Chaduc, S. Fouilloux, J.-C. Taveau, O. Lambert, M. Lansalot, E. Bourgeat-Lami, A. Thill, O. Spalla, S. Ravaine and E. Duguet, Polym. Chem., 2012, 3, 1130.

54 A. Perro, S. Reculusa, F. Pereira, M.-H. Delville, C. Mingotaud, E. Duguet, E. Bourgeat-Lami and S. Ravaine, Chem. Commun., 2005, 5542.

55 A. Perro, E. Duguet, O. Lambert, J. C. Taveau, E. Bourgeat-Lami and S. Ravaine, Angew. Chemie - Int. Ed., 2009, 48, 361.

56 J.-C. Taveau, D. Nguyen, A. Perro, S. Ravaine, E. Duguet and O. Lambert, Soft Matter, 2008, 4, 311.

57 A. Thill, A. Désert, S. Fouilloux, J.-C. Taveau, O. Lambert, M. Lansalot, E. Bourgeat-Lami, O. Spalla, L. Belloni, S. Ravaine and E. Duguet, Langmuir, 2012, 28, 11575.

58 A. Désert, J. Morele, J.-C. Taveau, O. Lambert, M. Lansalot, E. Bourgeat-Lami, A. Thill, O. Spalla, L. Belloni, S. Ravaine and E. Duguet, Nanoscale, 2016, 8, 5454.

59 C. Hubert, C. Chomette, A. Désert, M. Sun, M. TreguerDelapierre, S. Mornet, A. Perro, E. Duguet and S. Ravaine, Faraday Discuss., 2015, 181, 139.

60 X. G. Qiao, P.-Y. Dugas, B. Charleux, M. Lansalot and E. Bourgeat-Lami, Macromolecules, 2015, 48, 545.

61 I. Chaduc, J. Parvole, E. Duguet, S. Ravaine, M. Lansalot and E. Bourgeat-Lami, Polym. Chem., 2012, 3, 3232.

62 J. D. Forster, J.-G. Park, M. Mittal, H. Noh, C. F. Schreck, C. S. O'Hern, H. Cao, E. M. Furst and E. R. Dufresne, ACS Nano, 2011, 5, 6695.

63 E. Ducrot, M. He, G.-R. Yi and D. J. Pine, Nat. Mater., 2017, 16, 652.

64 P.-G. de Gennes, Angew. Chemie Int. Ed. English, 1992, 31, 842.

65 B. P. Binks, Curr. Opin. Colloid Interface Sci., 2002, 7, 21.

66 E. Passas-Lagos and F. Schüth, Langmuir, 2015, 31, 7749.

67 B. W. Longbottom and S. A. F. Bon, Sci. Rep., 2018, 8, 1.

68 Q. Chen, J. Yan, J. Zhang, S. C. Bae and S. Granick, Langmuir, 2012, 28, 13555.

69 A. Perro, S. Reculusa, S. Ravaine, E. Bourgeat-Lami and E. Duguet, J. Mater. Chem., 2005, 15, 3745.

70 J. Du and R. K. O'Reilly, Chem. Soc. Rev., 2011, 40, 2402.

71 M. Lattuada and T. A. Hatton, Nano Today, 2011, 6, 286.

72 J. Hu, S. Zhou, Y. Sun, X. Fang and L. Wu, Chem. Soc. Rev., 2012, 41, 4356.

73 A. Walther and A. H. E. Müller, Chem. Rev., 2013, 113, 5194.

74 J. Zhang, B. A. Grzybowski and S. Granick, Langmuir, 2017, 33, 6964.

75 T. Ogi, L. B. Modesto-Lopez, F. Iskandar and K. Okuyama, 
Colloids Surfaces A Physicochem. Eng. Asp., 2007, 297, 71.

76 Y. Xie, S. Guo, C. Guo, M. He, D. Chen, Y. Ji, Z. Chen, X. Wu, Q. Liu and S. Xie, Langmuir, 2013, 29, 6232.

77 A. B. Pawar and I. Kretzschmar, Langmuir, 2008, 24, 355.

78 S. Jiang, M. J. Schultz, Q. Chen, J. S. Moore and S. Granick, Langmuir, 2008, 24, 10073.

79 A. Perro, F. Meunier, V. Schmitt and S. Ravaine, Colloids Surfaces A Physicochem. Eng. Asp., 2009, 332, 57.

80 C. Huang and X. Shen, Chem. Commun., 2014, 50, 2646.

81 B. Liu, C. Zhang, J. Liu, X. Qu and Z. Yang, Chem. Commun., 2009, 3871.

82 S. Sacanna, W. T. M. Irvine, L. Rossi and D. J. Pine, Soft Matter, 2011, 7, 1631.

83 S. H. Kim, A. D. Hollingsworth, S. Sacanna, S. J. Chang, G. Lee, D. J. Pine and G. R. Yi, J. Am. Chem. Soc., 2012, 134, 16115.

84 A. B. Pawar and I. Kretzschmar, Langmuir, 2009, 25, 9057.

85 Z. He and I. Kretzschmar, Langmuir, 2013, 29, 15755.

86 Z. Nie, D. Fava, E. Kumacheva, S. Zou, G. C. Walker and M. Rubinstein, Nat. Mater., 2007, 6, 609.

87 B. Nikoobakht and M. A. El-Sayed, Chem. Mater., 2003, 15, 1957.

88 J.-H. Kim, H. J. Hwang, J. S. Oh, S. Sacanna and G.-R. Yi, J. Am Chem. Soc., 2018, 140, 9230.

89 L. Wang, L. Xia, G. Li, S. Ravaine and X. S. Zhao, Angew. Chemie Int. Ed., 2008, 47, 4725.

90 C. Bae, H. Kim, J. M. Montero Moreno, G.-R. Yi and H. Shin, Sci. Rep., 2015, 5, 9339.

91 H. Bao, W. Peukert and R. N. Klupp Taylor, Adv. Mater., 2011, 23, 2644.

92 H. Bao, T. Bihr, A.-S. Smith and R. N. Klupp Taylor, Nanoscale, 2014, 6, 3954.

93 A. Lotierzo, B. W. Longbottom, W. H. Lee and S. A. F. Bon, ACS Nano, 2019, 13, 399.

94 Y. Wang, Y. Wang, D. R. Breed, V. N. Manoharan, L. Feng, A. D. Hollingsworth, M. Weck and D. J. Pine, Nature, 2012, 491, 51.

95 Y. Wang, Y. Wang, X. Zheng, G. Yi, S. Sacanna, D. J. Pine and M. Weck, J. Am. Chem. Soc., 2014, 136, 6866.

96 X. Zheng, Y. Wang, Y. Wang, D. J. Pine and M. Weck, Chem. Mater., 2016, 28, 3984.

97 X. Zheng, M. Liu, M. He, D. J. Pine and M. Weck, Angew. Chemie Int. Ed., 2017, 56, 5507.

98 Z. Wang, Z. Wang, J. Li, S. T. H. Cheung, C. Tian, S.-H. Kim, G.R. Yi, E. Ducrot and Y. Wang, J. Am. Chem. Soc., 2019, 141, 14853.

99 Z. Gong, T. Hueckel, G.-R. Yi and S. Sacanna, Nature, 2017, 550, 234.

100 A. Désert, C. Hubert, Z. Fu, L. Moulet, J. Majimel, P. Barboteau, A. Thill, M. Lansalot, E. Bourgeat-Lami, E. Duguet and S. Ravaine, Angew. Chemie Int. Ed., 2013, 52, 11068.

101 Y. Huang, J. Wang, J. Zhou, L. Xu, Z. Li, Y. Zhang, J. Wang, Y. Song and L. Jiang, Macromolecules, 2011, 44, 2404.

102 R. Deng, H. Li, J. Zhu, B. Li, F. Liang, F. Jia, X. Qu and Z. Yang, Macromolecules, 2016, 49, 1362.

103 R. Deng, H. Li, F. Liang, J. Zhu, B. Li, X. Xie and Z. Yang, Macromolecules, 2015, 48, 5855.

104 K. H. Ku, Y. Kim, G.-R. Yi, Y. S. Jung and B. J. Kim, ACS Nano, 2015, 9, 11333
105 A. H. Gröschel, F. H. Schacher, H. Schmalz, O. V. Borisov, E. B. Zhulina, A. Walther and A. H. E. Müller, Nat. Commun., 2012, 3, 710 .

106 E. Bianchi, R. Blaak and C. N. Likos, Phys. Chem. Chem. Phys., 2011, 13, 6397.

107 S. Gangwal, A. Pawar, I. Kretzschmar and O. D. Velev, Soft Matter, 2010, 6, 1413.

108 J. Yan, M. Han, J. Zhang, C. Xu, E. Luijten and S. Granick, Nat. Mater., 2016, 15, 1095.

109 S. Sacanna, L. Rossi and D. J. Pine, J. Am. Chem. Soc., 2012, 134, 6112.

110 L. Rossi, in Frontiers of Nanoscience, Elsevier Ltd., 1st edn., 2019, vol. 13, pp. 1-22.

111 P. I. C. Teixeira and J. M. Tavares, Curr. Opin. Colloid Interface Sci., 2017, 30, 16.

112 C. Avendaño and F. A. Escobedo, Curr. Opin. Colloid Interface Sci., 2017, 30, 62.

113 L. Rovigatti, J. Russo and F. Romano, Eur. Phys. J. E, 2018, 41, 59.

114 S. Asakura and F. Oosawa, J. Chem. Phys., 1954, 22, 1255.

115 A. D. Dinsmore, A. G. Yodh and D. J. Pine, Phys. Rev. E, 1995, 52, 4045.

116 S. Sacanna, W. T. M. Irvine, P. M. Chaikin and D. J. Pine, Nature, 2010, 464, 575.

117 P.-E. Rouet, C. Chomette, E. Duguet and S. Ravaine, Angew. Chemie Int. Ed., 2018, 57, 15754.

118 P.-E. Rouet, C. Chomette, L. Adumeau, E. Duguet and S. Ravaine, Beilstein J. Nanotechnol., 2018, 9, 2989.

119 J. Zhang, E. Luijten and S. Granick, Annu. Rev. Phys. Chem., 2015, 66, 581.

120 L. Hong, A. Cacciuto, E. Luijten and S. Granick, Nano Lett., 2006, 6, 2510.

121 L. Hong, A. Cacciuto, E. Luijten and S. Granick, Langmuir, 2008, 24, 621 .

122 Q. Chen, J. K. Whitmer, S. Jiang, S. C. Bae, E. Luijten and S. Granick, Science, 2011, 331, 199.

123 D. J. Kraft, R. Ni, F. Smallenburg, M. Hermes, K. Yoon, D. A. Weitz, A. van Blaaderen, J. Groenewold, M. Dijkstra and W. K. Kegel, Proc. Natl. Acad. Sci., 2012, 109, 10787.

124 C. Yu, J. Zhang and S. Granick, Angew. Chemie Int. Ed., 2014, 53, 4364.

125 B. Bharti, D. Rutkowski, K. Han, A. U. Kumar, C. K. Hall and O. D. Velev, J. Am. Chem. Soc., 2016, 138, 14948.

126 H. Hu, F. Ji, Y. Xu, J. Yu, Q. Liu, L. Chen, Q. Chen, P. Wen, Y. Lifshitz, Y. Wang, Q. Zhang and S.-T. Lee, ACS Nano, 2016, 10, 7323.

127 N. Castro, D. Constantin, P. Davidson and B. Abécassis, Soft Matter, 2016, 12, 9666.

128 J. S. Oh, S. Lee, S. C. Glotzer, G.-R. Yi and D. J. Pine, Nat. Commun., 2019, 10, 3936.

129 T. S. Skelhon, Y. Chen and S. A. F. Bon, Soft Matter, 2014, 10, 7730.

130 X.-H. Ge, Y.-H. Geng, J. Chen and J.-H. Xu, ChemPhysChem, 2018, 19, 2009.

131 W. Li, S. Ravaine and E. Duguet, J. Colloid Interface Sci., 2020, $560,639$.

132 F. Guignard and M. Lattuada, Langmuir, 2015, 31, 4635. 
133 O. D. Velev and S. Gupta, Adv. Mater., 2009, 21, 1897.

134 K. Liu, N. Zhao and E. Kumacheva, Chem. Soc. Rev., 2011, 40, 656.

135 L. J. Hill, N. Pinna, K. Char and J. Pyun, Prog. Polym. Sci., 2015, 40, 85.

136 H. Wang, L. Chen, X. Shen, L. Zhu, J. He and H. Chen, Angew. Chemie Int. Ed., 2012, 51, 8021.

137 K. K. Caswell, J. N. Wilson, U. H. F. Bunz and C. J. Murphy, J. Am. Chem. Soc., 2003, 125, 13914.

138 D. Chandler, Nature, 2005, 437, 640.

139 J. Kim, C.-H. Choi, S.-J. Yeom, N. Eom, K.-K. Kang and C.-S. Lee, Langmuir, 2017, 33, 7503.

140 K. Liu, Z. Nie, N. Zhao, W. Li, M. Rubinstein and E. Kumacheva, Science, 2010, 329, 197.

141 Z. Nie, D. Fava, M. Rubinstein and E. Kumacheva, J. Am. Chem. Soc., 2008, 130, 3683.

142 K. Liu, A. Lukach, K. Sugikawa, S. Chung, J. Vickery, H. TherienAubin, B. Yang, M. Rubinstein and E. Kumacheva, Angew. Chemie Int. Ed., 2014, 53, 2648.

143 A. Klinkova, H. Therien-Aubin, R. M. Choueiri, M. Rubinstein and E. Kumacheva, Proc. Natl. Acad. Sci., 2013, 110, 18775.

144 K. Liu, C. Resetco and E. Kumacheva, Nanoscale, 2012, 4, 6574.

145 A. Lukach, K. Liu, H. Therien-Aubin and E. Kumacheva, J. Am. Chem. Soc., 2012, 134, 18853.

146 D. Fava, M. A. Winnik and E. Kumacheva, Chem. Commun., 2009, 2571.

147 G. Chen, K. J. Gibson, D. Liu, H. C. Rees, J.-H. Lee, W. Xia, R. Lin, H. L. Xin, O. Gang and Y. Weizmann, Nat. Mater., 2019, 18, 169.

148 T. Tigges and A. Walther, Angew. Chemie Int. Ed., 2016, 55, 11261.

149 Y. Wang, A. D. Hollingsworth, S. K. Yang, S. Patel, D. J. Pine and M. Weck, J. Am. Chem. Soc., 2013, 135, 14064.

150 E. Elacqua, X. Zheng and M. Weck, ACS Macro Lett., 2017, 6, 1060.

151 F. Benyettou, X. Zheng, E. Elacqua, Y. Wang, P. Dalvand, Z. Asfari, J.-C. Olsen, D. S. Han, N. Saleh, M. Elhabiri, M. Weck and A. Trabolsi, Langmuir, 2016, 32, 7144.

152 A. H. Gröschel, A. Walther, T. I. Löbling, F. H. Schacher, H. Schmalz and A. H. E. Müller, Nature, 2013, 503, 247.

153 J.-H. Kim, W. J. Kwon and B.-H. Sohn, Chem. Commun., 2015, 51, 3324.

154 S. Chae, S. Lee, K. Kim, S. W. Jang and B.-H. Sohn, Chem. Commun., 2016, 52, 6475.

155 S. Lee, S. Jang, K. Kim, J. Jeon, S.-S. Kim and B.-H. Sohn, Chem. Commun., 2016, 52, 9430.

156 Q. Chen, S. C. Bae and S. Granick, Nature, 2011, 469, 381.

157 F. Romano and F. Sciortino, Soft Matter, 2011, 7, 5799.

158 F. Romano and F. Sciortino, Nat. Mater., 2011, 10, 171.

159 G. Doppelbauer, E. Bianchi and G. Kahl, J. Phys. Condens. Matter, 2010, 22, 104105.

160 F. Romano, E. Sanz, P. Tartaglia and F. Sciortino, J. Phys. Condens. Matter, 2012, 24, 064113.

161 Z.-W. Li, Y.-L. Zhu, Z.-Y. Lu and Z.-Y. Sun, Soft Matter, 2016, 12 741.

162 E. G. Noya, N. G. Almarza and E. Lomba, Soft Matter, 2017, 13,
3221.

163 D. Chen, G. Zhang and S. Torquato, J. Phys. Chem. B, 2018, 122, 8462.

164 A. Kim, S. Zhou, L. Yao, S. Ni, B. Luo, C. E. Sing and Q. Chen, J. Am. Chem. Soc., 2019, 141, 11796.

165 Zhang, A. S. Keys, T. Chen and S. C. Glotzer, Langmuir, 2005, 21, 11547.

166 F. Smallenburg and F. Sciortino, Nat. Phys., 2013, 9, 554.

167 G. van Anders, N. K. Ahmed, R. Smith, M. Engel and S. C. Glotzer, ACS Nano, 2014, 8, 931.

168 N. Patra and A. V Tkachenko, Phys. Rev. E, 2018, 98, 032611.

169 W. Liu, M. Tagawa, H. L. Xin, T. Wang, H. Emamy, H. Li, K. G. Yager, F. W. Starr, A. V Tkachenko and O. Gang, Science, 2016, 351, 582. 